\section{Ruolo e salvaguardia delle evidenze stratigrafiche nel progetto e nel cantiere di restauro}

\author{
FrANCESCO DOGLIONI \\ Istituto Universitario d'Architettura di Venezia
}

\begin{abstract}
Riassunto
La relazione affronta diversi aspetti del rapporto tra stratigrafia e restauro architettonico. Dopo aver descritto la posizione nei confronti dell'utilizzo della stratigrafia delle principali correnti di pensiero sul restauro in Italia, vengono trattate la questione dell'autenticità e degli interventi a contrasto degli effetti di degrado che coinvolgono le tracce della stratificazione. Ai fini della riconoscibilità degli interventi di restauro, si propone di concepirli e realizzarli come strati intenzionalmente realizzati per consentire a posteriori la lettura della sequenza, e si indicano accorgimenti e modi operativi. Vengono presentati tre casi di restauro (il Chiostro delle Cucine nel Convento di S. Paolo a Parma, la chiesa di S. Marcello a Feltre, il Convento di S. Cosma e Damiano a Venezia) che, con modalità articolate, cercano di coniugare la conservazione e la leggibilità delle tracce con gli obiettivi più generali del restauro.
\end{abstract}

Parole chiave: Stratigrafia, Restauro Architettonico, Autenticità.

\begin{abstract}
The report deals with the relationship between stratigraphy and architectural restoration.

After illustrating the position towards the use of stratigraphy of the main currents of opinion on restoration in Italy, the report tackles the question of authenticity and of the interventions to minimise the deterioration effects involving the stratification traces. In order to ensure the recognizability of the restoration interventions, it is proposed to conceive and carry them out as deliberately developed layers, in order to allow the subsequent reading of the sequence. To this aim tricks and procedures are illustrated.

The cases of restoration are presented (the "Chiostro delle Cucine" in St. Paul Convent in Parma, St. Marcellus Church in Feltre and St. Cosma and St. Damiano Convent in Venice), which, by means of elaborated techniques, try to combine the conservation and readability of the traces with more general tasks of the restoration.
\end{abstract}

Key words: Stratigraphy, Architectural Restoration, Authenticity.
Per affrontare l'argomento del ruolo e della salvaguardia delle tracce visibili della stratificazione dobbiamo chiederci quale significato il restauro attribuisca oggi alla storia costruttiva, obiettivo dello studio stratigrafico, e più in generale ai segni del passaggio del tempo, tra i quali gli effetti del degrado.

N ella prima parte di questo scritto svolgerò alcune riflessioni sull'impostazione concettuale del progetto di restauro, per capi re qual e spazio possa essere attribuito al la stratificazione costruttiva; nella seconda proporrò al cuni interventi che hanno affrontato questo tema, per discuterne l'impostazione e i principali aspetti applicativi.

La ricognizione stratigrafica negli ultimi anni è più di frequente inclusa tra le analisi preliminari al restauro, rivol ta alle sole superfici visibili, oppure collegata a saggi stratigrafici nel suolo 0 in el evato per accertare la potenzial ità di depositi o la natura di assetti sottostrato.

Essa è divenuta gradualmente parte delle forme di conoscenza su cui si ritiene che il progetto di restauro debba fondarsi e di cui debba tenere conto, insieme allo studio e alla diagnosi dei fenomeni di degrado e di dissesto.

A questa generica convinzione non corrisponde uno sviluppo dei modi con cui il progetto di restauro possa realmente trarre utilità e fondamento dalla conoscenza della stratificazione e dei segni che la rendono osservabile, ol tre che dalle conseguenti interpretazioni storico-costruttive. Si comprende bene che, mentre la funzionalità degli studi sul degrado e sul dissesto è chiara, in quanto indagano sulla natura e sull'evoluzione dei fenomeni da contrastare per la conservazione dell'opera, il ruol o della stratigrafia è più complesso, perché la conoscenza archeologica cui essa contribuisce è di tipo storico: il suo ruolo nel progetto va cercato perciò nel delicato asse tra storia e restauro, tra conoscenza storica e restauro architettonico. II rapporto tra storia e restauro è da tempo dibattuto, e a partire dai diversi modi di concepirlo si sono verificate le più forti divaricazioni nella disciplina; è perciò inevitabile che anche il contributo della stratigrafia al progetto sia controverso.

Se mi è consentita una schematizzazione, in Italia le posizioni riferite al restauro critico, forse anche a seguito di una negativa presa di posizione da parte di Renato Bonelli negli anni '80 (Bonelul, 1986: 5-10), alla stratigrafia come strumento di conoscenza hanno continuato a preferire analisi costruttive e storico-archivistiche condotte secondo metodi già consolidati. Di conseguenza, la posizione che assume come centrale nel restauro il giudizio critico basato sulla conoscenza storica non ha fino ad oggi incluso organicamente la stratigrafia tra i 
propri strumenti, pur dimostrando per essa un interesse scientifico.

Sal vo al cune eccezioni e pur senza partire da negazioni di principio, non dimostrano particolare attenzione per il metodo stratigrafico nemmeno le posizioni in varia misura e modo riconducibili al ceppo del restauro scientifico, al quale possono essere riferiti numerosi interventi sulla stratificazione costruttiva real izzati in particol are da enti di tutela.

$\mathrm{N}$ ell'ambito della concezione conservativa, invece, diversi studiosi hanno indirizzato il proprio interesse allo sviluppo della stratigrafia come strumento di conoscenza. Tuttavia, la scelta di non applicare all'opera forme di giudizio selettivo basate su interpretazioni storiografiche (BeLLIN I, 1983), scelta che costituisce il nocciolo della differenza tra conservazione e restauro e vuol e sancire la separazione tra esiti della conoscenza storica e progetto, viene estesa ora anche al la stratigrafia, della quale si riconosce il potenziale conoscitivo, ma che si ritiene comunque non debba influenzare il restauro, se non rafforzando la scelta di conservare la materia costruita per la maggiore consapevol ezza del patrimonio di conoscenze in essa contenuto. Per questi studiosi, la preoccupazione che la stratigrafia possa essere utilizzata per forme di giudizio sel ettivo o per interventi di ricostruzione ripristino, ne preclude un più articolato ed esplicito impiego nel progetto (TreCCANI, 2000: 11-96).

II quadro italiano su questo argomento appare quindi stranamente asimmetrico e bloccato, in quanto chi più pratica la stratigrafia come strumento analitico non intende estendernel'utilizzo nel progetto, mentre chi in linea di principio potrebbe essere aperto a coinvolgerne gli esiti nel progetto non la pratica diffusamente.

Se accettiamo che la stratificazione sia un dato costitutivo che caratterizza il patrimonio architettonico, dobbiamo inevitabilmente coinvol gere la sua conoscenza nel progetto, tenendo conto che l'assenza di un modo sistematico per includerla e trattarla nel restauro amplia lo spazio di altri obiettivi e tecniche che non sempre hanno per il patrimonio costruito speciali attenzioni conservative. Per questo da tempo studio e sperimento modi operativi con cui, nell'assumere le scel te comunque necessarie del progetto di restauro, tenere conto in modo responsabile del la stratificazione attraverso l'impiego della stratigrafia, cercando fondamenti in essa e mutuandone in parte gli strumenti.

II restauro ha come fine principale e generalmente riconosciuto il prolungamento dell'esistenza dell'opera. Si ritiene fondamentale che in questo passaggio nel tempo
I'opera mantenga la propria autenticità, e per questo motivo molti studiosi considerano fattore essenziale la conservazione della materia di cui è costruita. La stratigrafia indaga le relazioni esistenti tra le parti materiali dell'edificio, e quindi costituisce un indicatore molto sensibile ai loro mutamenti, in grado di registrare le perdite di leggibilità verificabilità, le sostituzioni material i o le al terazioni rilevanti, fattori che contribuiscono al la progressiva perdita di autenticità.

Sintetizzo queste considerazioni in una struttura logica che chiamo sill ogismo principal e:

- se il restauro ha come fine primo (in alternativa: come condizione) la conservazione del l'autenticità del'edificio;

- se attribuiamo alla stratificazione il ruolo di condizione costitutiva edi struttura relazionale del l'autenticità, in quanto costituisce un sigillo reciproco tra le parti dell'edificio, le qualifica e le pone in relazione rispetto al tutto (DOGLIONI, 1993);

- allora la conservazione del la stratificazioneèun fine primo (in al ternativa: una condizione obbligata) del restauro.

Se accettiamo questo, ammettiamo di conseguenza che la stratigrafia possa essere utile al progetto per governare e controllare i mutamenti che incidono sull'autenticità, allo scopo di ridurne l'entità e l'impatto. Perciò ammettiamo che la stratigrafia possa:

- segnal are al progetto di restauro la struttura rel azionale conosciuta dell'edificio, le superfici e i nessi da cui sono stati tratti i dati fondamentali; il progetto di conseguenza può prevedere specifiche attenzioni conservative per queste parti a maggiore densità;

- verificare l'impatto che le diverse operazioni di cui si compone il progetto, anche di natura conservativa, produrranno sulla stratificazione, e introdurre correttivi per mitigare i danni conseguenti;

- indirizzare le attività di cantiere verso una specifica attenzione conservativa, riducendo il rischio di danni involontari ai segni fondamentali, spesso prodotti dagli interventi strutturali e impiantistici;

- verificare al termine dell'intervento l'entità e la natura delle modificazioni prodotte, e l'allontanamento dalla condizione inizial el.

L'attribuire significato e attenzioni conservative alle tracce della storia costruttiva rappresenta solo uno tra

${ }^{1} \mathrm{G}$. Cristinelli, a questo proposito, pone quello che definisce "... il principio fondamentale del restauro: quello per il quale (...) un edificio non può diventare un'al tra cosa dopo aver subito un intervento, per I'appunto, di restauro. Si sa che le cose mutano nel corso del tempo, ma si sa anche che al cune rimangono sè stesse nella loro sostanza pur nel mutamento. Qual è il limite della mutazione oltre il quale gli edifici perdono la loro sostanza e diventano al tro da sè?" (CRISTINELLI, 2000: 25) 
gli obiettivi che il restauro può includere, ma che non sempre include, obiettivi che possono spaziare, solo per citarne alcuni, dalla conservazione strutturale e materica, al restauro della configurazione architettonica, agli adattamenti per l'utilizzo compatibile. Per affrontare le scelte conflittuali che si pongono al voler perseguire con uno stesso progetto obiettivi eterogenei e diversamente misurabili, diventa necessario non tanto stabilire in via definitiva e astratta gerarchie, pesi o priorità tra di essi - ritengo ardua e poco produttiva questa strada - quanto, dopo aver messo a fuoco i singoli obiettivi, ricercare sul piano progettuale ed operativo le possibili soluzioni e gli accorgimenti per una loro composizione. Questo significa, dopo una prima riflessione, passare dal piano teorico a quello della ricerca pratica e duttile dei modi con cui attuare gli obiettivi di un dato progetto su un edificio, esaminando in concreto le soluzioni capaci di contemperare i diversi requisiti richiesti, per ritornare poi sul piano dei principi e degli obiettivi generali del restauro, sulla scorta dei quali verificare criticamente e affinare la reciproca compatibilità degli interventi ipotizzati. II percorso può ovviamente essere ripetuto più volte.

Un tale metodo non può perciò essere esclusivamente deduttivo (dai principi generali alle applicazioni, dalla teoria alla prassi), ma deve seguire un percorso a tratti induttivo e a tratti deduttivo, e questo rende concettualmente necessaria la presenza sia dell'operatività che del singolo caso reale incluso in una casistica più vasta anche per sviluppare a fondo le questioni di principio. Vi è quindi una reciproca necessità: se le general izzazioni e le scelte a priori non sono produttive, in quanto per realizzarsi devono accettare il confronto con i casi reali, tuttavia per affrontare il singolo caso servono i principi generali alla luce dei quali affinare le soluzioni fino a giudicarle accettabili e ad operare la scelta.

II tema che affrontiamo deve comunque essere sal damente ancorato ad una concezione complessiva dell'opera di restauro, e non può essere risol to separatamente: non credo possa o debba esistere un restauro stratigrafico, anche se vi sono casi in cui l'intervento deve accentuare l'attenzi one per questo aspetto rispetto ad al tri obiettivi. Di sicuro la stratigrafia costituisce una angolazione particolare dalla quale riflettere sul restauro e sui suoi esiti.

Tuttavia la nostra società e la nostra cultura chiedono con forza all'opera di restauro:

- I'arresto definitivo delle cause di degrado;

- Ia restituzione di uno "stato di decoro", che spesso viene interpretato come la cancellazione totale degli effetti di degrado e il ripristino di una condizione genericamente precedente o compiuta, che sfocia sovente nel rinnovo;

- il conferimento di requisiti idonei all'uso modernamente inteso, (adattamento ad un nuovo uso o prosecuzi one di un uso storico con standards rinnovati) con gli adattamenti strutturali, formali, impiantistici e funzionali conseguenti;

- il soddisfacimento di aspettative di val orizzazione a fini simbolici, economici, turistici, ecc.

Tutto questo, nel migliore dei casi, genera l'idea diffusa di restauro come categoria allargata, il cui senso si amplia ben al di fuori dell'orbita disciplinare ela cui soluzione viene spesso ricercata nel più vasto campo della progettazi one architettonica.

$\mathrm{Ma}$ anche come categoria ristretta, ossia nella zona meglio controllata del recinto disciplinare, ol tre alla trasmissione al futuro e al mantenimento dell'autenticità il restauro si pone altri fini, non sempre accettati da tutti gli studiosi, fini che possiamo condensare nella messa in valore delle peculiarità (storiche, architettoniche, artistiche, antropologiche, ecc.) dell'opera, attraverso una valutazione critica.

Azioni molto diverse, spesso in combinazione tra Ioro, possono proporsi questo fine:

- interventi a contrasto degli effetti di degrado, come ad esempio la pulitura, per ridurne il disturbo visivo e restituire decoro e condizioni visuali migliori;

- interventi di messa in luce di superfici, anche attraverso rimozioni di strati sovrapposti, o di recupero di assetti spaziali antichi, attraverso demolizione di elementi divisori;

- interventi di integrazione di lacune, per ridurre la frammentarietà;

- interventi di ricostruzione di intere parti, per raggiungere forme di unitarietà perdute.

A complicare la materia, alla eterogeneità degli obiettivi normalmente inclusi nel restauro si somma la singolarità di ogni edificio: ciascuno ha una storia e un carattere diverso, cui contribuiscono il numero e la qual ità dei diversi assetti, lo stato di degrado, la leggibilità materica, il grado di unitarietà, la "forza" dell'ultimo assetto rispetto ai precedenti, ecc. Per avvicinarci al nostro argomento, possiamo tentare di addensare schematicamente due gruppi di situazioni:

- nel primo gruppo vi sono gli edifici nel cui carattere preval e il ruolo della matericità, della stratificazione degli assetti, del degrado come segno del passaggio del tempo. In questi edifici la stratigrafia è in genere applicabile con maggiore efficacia ed organicità; 
- nel secondo gruppo, degrado, stratificazione e materia sono sullo sfondo o in filigrana rispetto all'unitarietà della configurazione architettonica, nella quale un assetto prevale largamente su altri eventualmente esistenti, all'omogeneità delle superfici o altro ancora. In questo secondo gruppo, la stratigrafia incontra maggiori difficoltà applicative e giunge a risultati che possono pur sempre essere significativi, ma in ogni caso sono del tutto parziali.

E' evidente come esista una ampia zona di transizione tra i due gruppi, e come questi possano essere ulteriormente articolati al loro interno. Inol tre molti edifici sono costituiti da parti riconducibili ora al primo ora al secondo gruppo, formando un insieme eterogeneo: vedi ad esempio il caso di seguito descritto del chiostro di $\mathrm{S}$. Paolo a Parma.

Voglio rimarcare come vi sia un rapporto spesso biunivoco tra il carattere della costruzione, schematicamente riconducibile ad uno di questi due gruppi, e le condizioni di leggibilità stratigrafica. Gli edifici riconducibili al primo gruppo si prestano maggi ormente al la lettura stratigrafica.

Per esprimere una prima opzione di indirizzo progettual e, ritengo che il restauro debba cercare come primo riferimento questo carattere, senza forzare la costruzione a mutarlo radicalmente, e tenere in gran conto le condizioni di partenza per apportare correttivi parziali. N e consegue che, per compiere un intervento appropriato al carattere, il progetto di restauro deve mirare a non far passare l'edificio dal primo al secondo gruppo, o viceversa. Se questo passaggio non avviene, in particolare dal primo al secondo gruppo, vi sono anche le condizioni per mantenere una maggiore leggibilità stratigrafica.

Va ricordato che la stratigrafia della costruzione si applica in larga preval enza alle superfici visibili. Come già detto, alcuni fattori, come gli effetti del degrado, in genere accentuano la leggibilità stratigrafica delle superfici, con azioni erosive e comportamenti differenziali. Ogni azione che punti a sostituire e rinnovare sistematicamente le superfici, anche per cancellare effetti di degrado, ha in genere un el evato impatto stratigrafico, ossia distrugge 0 rende inaccessibili i dati in base ai quali la stratigrafia è possibile. Se la costruzione si riduce ad una unica unità riconoscibile, la stratigrafia ha senso solo per constatare I'avvenuto mutamento, con le ripercussioni sull'autenticità prima citate.

Q uesto induce ad accentuare l'attenzione conservativa sulle superfici, e ad assumere nei confronti degli effetti di degrado un indirizzo di attenuazione dei segni, piuttosto che di cancellazione (TorseLLO, 1988: 23-31), in quanto il primo indirizzo può essere compatibile con il mantenimento dei dati stratigrafici, certo non il secondo. In via generale, appare chiara l'importanza di ridurre le alterazioni e le sostituzioni sistematiche di superfici.

Questo ha una rilevante implicazione riguardo al trattamento nel progetto degli effetti di degrado, cui spesso indirettamente si deve la visibilità della stratificazione costruttiva, implicazione che schematizzerò cos̀:

Sillogismo n. 1

- spesso le tracce della stratificazione costruttiva sono state riportate "naturalmente" in luce dagli effeti de degrado differenziale del le superfic;

- in questi casi, effetti del degrado e tracce della stratificazione non sono separabili e costituiscono evidenze stratigrafiche fortemente compenetrate;

- allora, se in questi casi voglio conservare le tracce strati grafiche e la loro leggibilità, non posso cancellare de tutto gli effeti del degrado, ma devo dedicare attenzione ad arrestare le cause di degrado (conservandone gli effetti), o intervenire anche sugli effetti sol tanto attenuandoli.

E' evidente come una simile impostazione contrasti sia con gli obiettivi di ordine estetico-architettonico posti da al cune posizioni disciplinari del restauro, sia a maggior ragione con la concezione allargata del restauro, propria del senso comune. E' una strettoia mol to difficile da affrontare e gli esiti più diffusi sono sotto gli occhi di tutti.

Sovente succede che, dopo aver cercato di svolgere I'analisi stratigrafica su una costruzione o una sua parte, dobbiamo desistere perché l'edificio è stato restaurato, e questo si è tradotto o nel rinnovo delle superfici o in una diffusa alterazione causata da piccoli e grandi rifacimen$\mathrm{ti}$, a volte intenzionalmente mascherati, che confondono tra loro tutte le diverse superfici di strato, sfuocando o cancellando i bordi di contatto. Niente ci appare più riconoscibile con sicurezza. E' sal utare aver provato al meno una volta questa frustrazione come cultori di stratigrafia, in quanto ci spinge come restauratori a tentare di consentire ad altri in futuro quello che a noi è stato impedito.

Per ridurre il rischio di giungere anche involontariamente a questo risultato negativo, la stratigrafia suggerisce anche alcune regole, o più semplicemente accorgimenti pratici, che aumentano la riconoscibilità dell'aggiunta o dell'integrazione rispetto al contesto in cui è inserita e la spingono ad una maggiore nitidezza, contribuendo quindi a soddisfare il requisito proprio del restauro di rendere distinguibile la parte aggiunta, sia essa l'integrazione di una lacuna, un intervento strutturale o di al tra natura. 
Tra questi accorgimenti:

- non alterare o coprire completamente i bordi e le interfacce negative presenti nel punto di contatto tra nuovo e antico;

- ridurre il più possibile la formazione di nuovi bordi, preferendo la formazione di fal si bordi (bordi accostati che derivano la loro forma dalla parte preesistente).

II progetto e il cantiere di restauro devono perciò prevedere modi grafici e accorgimenti organizzativi per trasmettere agli operatori precise istruzioni.

Possiamo anche qui sintetizzare questo ragionamento in forma di sillogismo:

Sillogismo n. 2

- Se le trasformazioni de passato sono oggi riconoscibili attraverso la stratigrafia in quanto realizzate come stratificazioni (strati ed interfacce);

- se il restauro vuole rendere riconosabili in futuro le proprie azioni;

- allora le azioni di restauro possono essere applicate sulla fabbrica come stratificazioni intenzionali (strati ed interfacce), e la stratigrafia diviene strumento per la distinguibilità futura deleazioni di restauro.

Si apre perciò la strada all'utilizzo nel progetto del metodo e della mentalità stratigrafica al fine di rendere più nitida la riconoscibilità futura degli interventi positivi e negativi che compongono I'opera di restauro, attraverso una "deposizione-costruzione" che tenga conto di quali sono i fattori ele condizioni in grado di permettere il riconoscimento stratigrafico di un processo avvenuto. Va rimarcato che, mentre la cultura del restauro ha el aborato principi, metodi e soluzioni di riferimento per l'integrazione di lacune di manufatti ad al ta figuratività, concentrando su questi la propria attenzione, l'intervento di apporto sulle parti solo costruite è proporzional mente molto più trascurato, anche se rappresenta il campo di intervento quantitativamente preponderante.

La stratigrafia può essere utilizzata come strumento per riconoscere assetti precedenti all'ultimo visibile, anche associandosi a saggi, o per riconoscere dalle tracce l'esistenza in antico di parti ora perdute, e a volte la loro forma e dimensione. La conoscenza cos̀ raggiunta può essere utilizzata come base per realizzare integrazioni, siano esse volte a ricostruzioni intese tout-court al ripristino, pratica tanto antica quanto contrastata del restauro, 0 ad al tre più articol ate e misurate forme di reintegrazione.

Possiamo addensare due casi di segno contrario: nel primo, rimuovendo strati sovrapposti o divisioni funzionaIi, si rimettono in luce assetti costruttivi, decorativi o spaziali che riteniamo di el evata qualità e di completezza tal e da non richiedere poi, a loro volta, vasti interventi integrativi. Se ammettiamo la possibilità di esprimere un giudizio che comporti una sel ezione, quindi una demolizione di parti, possibilità che a determinate condizioni personalmente ammetto, questo caso può rientrare nella val orizzazione de caratteri peculiari di un edificio. Deve essere chiaro in ogni momento cosa si perde e a favore di che cosa, e quindi I'azione distruttiva deve essere ponderata (cosa si perde, cosa si acquisisce, quali ulteriori interventi si rendono necessari), progettata e documentata anche stratigraficamente. Ove possibile, si può prevedere di mantenere sull'opera tracce dell'avvenuta demolizione (interfacce negative, testimoni di vario tipo).

$\mathrm{N}$ el secondo caso, a partire da interfacce negative, si ricostruisce 0 si integra una parte perduta. $\mathrm{N}$ on ritengo che questa scel ta debba essere accol ta o rifiutata in linea di principio, ma vada operata dopo una attenta valutazione dei fattori che concorrono nel singolo caso, con in più una richiesta della stratigrafia al restauro: che non sia distrutta o danneggiata la traccia su cui si basa l'ipotesi ricostruttiva prima e la ricostruzione poi, ma sia conservata e mantenuta visibile in modo da rendere ripetibile il processo interpretativo in base al qual e è stata assunta la decisione. Questo comporta, ad esempio, adattare a falso bordo la parte che si vuole ricostruire all'interfaccia negativa che testimonia l'antica demol izione, mantenendone la riconoscibilità nel punto di contatto. A nche le tecniche di connessione tra la parte antica e quella nuova devono essere opportunamente scelte ed adattate, in quanto alcune presuppongono un impatto total mente distruttivo sulla traccia da conservare, e quindi dovrebbero essere escluse.

Se non ritengo si possa attribuire ad uno strumento la responsabilità di un eventuale utilizzo forzato, improprio o semplicemente non condivisibile dei suoi esiti, si deve tuttavia chiedere che sia utilizzato con coerenza, a parti re proprio dalla conservazione fisica delle tracce.

Sin qui, ritengo di aver portato alcuni argomenti a favore dell'inserimento organico delle tecniche e della mental ità stratigrafica nel processo di ideazi one e realizzazione del restauro. Prima di iniziare la seconda parte, rivol ta alla presentazione e discussione di casi con cui ho cercato di dare attuazione ai principi esposti, vorrei ricordare in sintesi alcune particolarità o, se preferiamo, accentuazioni tra il percorso di rilevamento e interpretazione dei dati stratigrafici per le costruzioni cosi come da me proposto alcuni anni fa (Doglioni, 1988: 223-247; DOGLIONI, 1997) e il metodo diffusamente accettato (PARENTI, 1985: 417-437; Brogiolo, 1988), maggiormente aderente al la stratigrafia applicata allo scavo. Premetto 


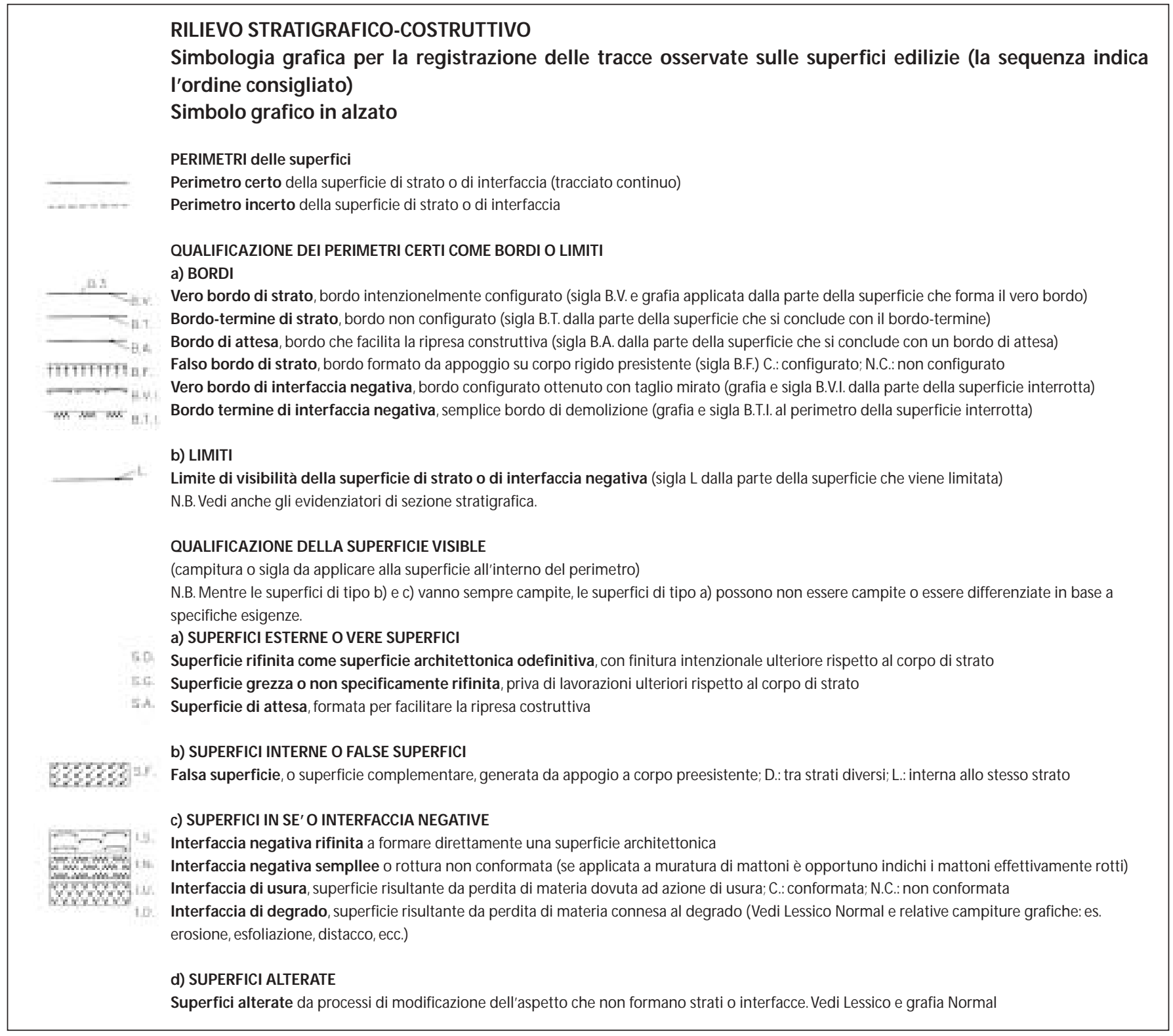


che io stesso ovviamente condivido tale metodo ma, come ho cercato di spiegare, constato alcune difficol tà:

- la leggibilità stratigrafica di un edificio dipende dalle sue superfici e dai contatti al perimetro tra superfici diverse; essa è perciò necessariamente parziale, e dipende in larga misura dalla presenza o meno di strati di rivestimento, dal degrado e dal dissesto, ossia da fattori che possono ridurre 0 accentuare la visibilità delle superfici e delle discontinuità;

- le diverse tracce di stratificazione presenti e visibili su superfici e perimetri, tracce che rappresentano solo una quota, a vol te piccola, rispetto al total e delle superfici, non hanno tutte la stessa evidenza e capacità di risoluzione; al cune sono chiare al punto che la loro lettura immediata ed istintiva viene confermata dall'esame razionale e obiettivo, con nulle o limitatissime possibilità di trovare argomenti a favore di una interpretazione diversa. In questi casi si può affermare che la traccia è un dato stratigrafico. Altre invece sono incomplete, dubbie quando non apparentemente contraddittorie, e la quota interpretativa diventa via via maggiore, dando più spazio ad argomenti contrari. Ritengo che la diversa evidenza e capacità di risoluzione delle tracce fisiche debba essere registrata prima di attribuire tout-court il ruolo di dato stratigrafico, in modo da segnalare la presenza di una più o meno forte componente interpretativa;

- se è vero che i dati non sono equipotenziali, ossia non hanno la stessa risoluzione ed evidenza ai fini strettamente stratigrafici, va anche aggiunto che alcuni di essi hanno una "componente narrativa" maggiore di altri: non mi dicono solo quale parte sia precedente e quale successiva, ma, ad esempio, come fosse formata una finestra poi tamponata, o rifinita una superficie poi ricoperta. Q uindi apportano dati preziosi anche ai fini della lettura della configurazi one e qual ificazi one architettonica dell'edificio nel tempo. Vi è il rischio, nella tensione prevalente a ricostruire la sequenza nella matrice di $\mathrm{H}$ arris, di perdere parole importanti del racconto (e a questo rischio il restauro deve essere molto attento); ma vi è anche il rischio, omettendo al cuni aspetti qualitativi della traccia, di ridurne la risoluzione strettamente stratigrafica: un intonaco che riveste uno spigolo cui si è affiancata una muratura documenta si una intenzione di configurazione e finitura importanti per la storia della fabbrica, ma rafforza anche l'interpretazione della sequenza fino a renderla indiscutibile.

Per questi motivi, ho proposto che la redazione delI'analisi stratigrafica sia maggiormente basata sulla caratterizzazione qualitativa dei margini della superficie visibi- le nei punti di discontinuità e di contatto con una superficie diversa di strato. Opero perciò una mappatura dei diversi tipi di bordo (bordo vero intenzionale, bordo falso 0 appoggiato, bordo di attesa, bordo-termine), di interfaccia (a taglio mirato o taglio andante) o di limite di visibilità, e una corrispondente qualificazione delle superfici segnando i dati osservati con grafismi convenzionali applicati ai rilievi o, meglio, al fotopiano della superficie esaminata (fig. 1). Indico anche i perimetri che non riesco ad interpretare perché confusi, al terati o seminascosti, o in cui permangono dei dubbi. Questi segni grafici, applicati separatamente sui margini di entrambe le superfici lungo la linea di discontinuità che ne forma il perimetro, vengono utilizzati per registrare cosa ritengo di riconoscere nella traccia che ho di fronte, in base alla mia biblioteca mentale di tracce. Si tratta quindi di una lettura stretta del singolo dato locale, che non esclude ma confina la quota di interpretazione soggettiva, e che precede e sostiene con i propri contenuti gli sviluppi interpretativi successivi, prevalentemente contestuali e di insieme: la sequenza relativa tra due superfici a contatto, il riconoscimento delle unità stratigrafiche, la formazione della sequenza complessiva e delle fasi, ecc.

Cerco di dividere la raccolta dei dati dalla loro interpretazione, anche se sono consapevole che questo è solo parzial mente possibile.

A mio avviso, questo procedimento presenta il vantaggio di costruire progressivamente l'interpretazione stratigrafica, basandola sulla combinazione di dati puntuaIi al contatto tra superfici, e di renderla maggiormente verificabile e de-costruibile (Popper avrebbe detto "fal sificabile", inteso come requisito dell'indagine scientifica) nel caso emergano ulteriori dati o sia effettuata una lettura diversa di alcuni di essi.

$\mathrm{Ma}$, e sposto le argomentazioni dal campo analitico della stratigrafia a quel lo conservativo, la mappatura delle tracce cosi condotta presenta indubbi vantaggi pratici ai fini della loro conservazione nel progetto di restauro, ed è questo il motivo principale che mi ha indotto a proporla e praticarla.

Infatti, ritengo che nel progetto debbano essere rispettate il più possibile le tracce portatrici di dati, non tanto la loro interpretazione, che per essere ancora ripetibile e verificabile ad intervento concluso deve basarsi sui dati leggibili nella materia. Sappiamo che questo è di fatto pressochè impossibile, perchéogni intervento, anche il più attento elimitato, introduce modificazioni fisiche o di leggibilità. $\mathrm{Ma}$, come già detto, le tracce non sono distribuite sulla fabbrica in modo equipotenziale, e mentre alcune 
hanno una elevatissima capacità di risoluzione, a volte concentrata in limitati punti di contatto tra unità, altre sono già confuse e risultano illeggibili senza ricorrere a forti contributi interpretativi. E' necessario che le tracce fondamental i vengano conservate attentamente, e perciò la mappatura dei segni al contatto tra unità si presta a diventare la "mappa dei segni da proteggere". II che non significa abbandonare al proprio destino le tracce incerte e illeggibili: queste, tral'al tro, potranno essere ulteriormente indagate proprio durante l'azione di restauro. Ma di certo dobbiamo scongiurare il rischio che i caposaldi ai quali si appiglia la nostra possibilità di riconoscere il processo costruttivo, spesso formati da un numero limitato di segni, siano compromessi o distrutti in modo anche involontario dalle azioni fisiche che accompagnano il restauro.

Se, invece, la mappatura delle unità stratigrafiche, estesa a tutte le superfici visibili dell'edificio, non qualifica e segnala in modo più marcato i punti di distinzionecontatto tra unità e le tracce fondamentali, si giunge ad una equipotenzial ità conservativa condivisi bile in linea di principio, ma difficilmente sostenibile e praticabile nella gran parte dei casi, con la conseguenza di aumentare le possibilità che ad essere col piti siano proprio i punti maggiormente significativi, perché su di essi non si è concentrata sufficientemente l'attenzione.

A questo proposito propongo il sillogismo n.3:

\section{Sillogismo n. 3}

- le tracce fisiche sulle quali si fonda la stratigrafia non sono equi potenziali, in quanto vi sono parti o punti (in genere bordi e interfacce) in cui si concentra la maggiore risoluzione e potenzialità informativa;

- se anche io conservo complessi vamente l'edificio, ma distruggo 0 nascondo questo tipo di tracce cardine, ho una drastica perdita di leggibilità stratigrafica;

- perciò devo salvaguardare in modo speciale le tracce ad alta potenzialità informativa, ad esempio formando una mappa dé segni da proteggere in modo particolare, ponendo segnali sul posto in fase di cantiere, ecc.

In sostanza, mi interessa che l'interpretazione sia ancora possi bile dopo il mio intervento a partire dal le tracce che ho riconosciuto come fondamentali, non omologare I'edificio restaurato alla mia lettura stratigrafica, o riproporre gli esiti di questa sulle pareti, formando su di esse una sorta di mappatura didascalica o di diagramma. Per questo, le tecniche moderne ci consentono di realizzare immagini virtuali la cui visione di fronte all'opera è certamente efficace, risparmiando all'edificio manomissioni non necessarie.
A fianco di questa motivazione-conservare in modo mirato le tracce per rendere ripetibile l'anal isi el'interpretazione stratigrafica- ve ne è un'altra che riguarda il nostro rapporto emotivo con I'architettura. La traccia ha in sé una capacità testimoniale, e possiamo osservarla con gli intenti razionali e analitici che abbiamo descritto. M a accanto a questa, sovrapposta e fusa, vi è una capacità evocativa che agisce sulla nostra emozione.

Emozionarsi per un'interfaccia? Mi rendo conto che ciò può far sorridere.

Le evidenze delle trasformazioni avvenute consentono immediatamente e istintivamente di comprendere che sull'edificio è passato il tempo, scandito da eventi che lo hanno diviso in tanti intervalli prima/dopo. E' dunque un tempo segmentato, non più unico. A loro volta, ciascuna traccia allude più o meno esplicitamente ad una forma diversa, che la nostra mente in modo istintivo insegue ed immagina ben prima di mettere in moto la capacità razionale. Per mezzo di queste tracce l'edificio attira il nostro istinto all'esplorazione e alla conoscenza, e anche per mezzo dello stimolo all'immaginazione che inducono percepiamo l'aura dell'edificio, qui intesa come versante emozionale dell'autenticità (Quen DoLo, 2000); Ia loro possibile perdita incide su di essa quanto, come già detto, sulI'autenticità razional mente intesa.

Qual estrategia complessiva? Ci si pone, come restauratori che attribuiscono importanza alla stratificazione costruttiva e come cultori della stratigrafia, un doppio obiettivo:

- le tracce vanno in primo luogo salvaguardate, ne va il più possibile evitata la distruzione sia intenzionale che involontaria;

- il trattamento delle tracce di stratificazione può e deve avvenire entro un progetto animato da una poetica che rispetti il carattere dell'edificio; il trattamento deve essere il più possibile organico, ossia contrario al modo disorganico, episodico, selettivo, ostensivo di singoli elementi o brani separati dal contesto, (voyerismo stratigrafico) con cui sovente vengono presentate le preesistenze;

- dobbiamo cercare di ridurre il più possibile le conseguenze stratigrafiche delle azioni di restauro, ossia mitigare anche sotto il profilo stratigrafico l'insieme di impatti che sono conseguenti al restauro come riutilizzo, come consolidamento, ma anche come conservazione materica.

Dato che la nostra attenzione si concentra sulle superfici, possiamo proporre i seguenti indirizzi eaccorgimenti operativi: 
- Se l'obiettivo è di raggiungere un maggior decoro, mutare l'aspetto della superficie, non la materia, con puliture mirate, velature non coprenti che virano il colore senza occultare il dato tessiturale della materia, che rimane perciò riconoscibile;

- evitare per quanto possibili gli interventi generalizzati e omogeneizzanti, quali manipolazioni o ricoperture complessive delle superfici, sostituzioni complete di intonaco, rifugature complete dei giunti di malta;

- studiare modi mirati per contrastare il degrado e il dissesto, evitando impatti general izzati e salvaguardando comunque i punti e le tracce a maggior potenziale stratigrafico (vedi mappa dei segni da proteggere);

- non coprire mai i bordi di interfaccia negativa o i falsi bordi;

- ridurre la formazione di nuovi bordi;

- cercare di lasciare l'edificio in uno stato "natural e" non troppo elaborato o concettual izzato;

- cercare di rispettare il carattere acquisito nel tempo dall'edificio, senza mutarlo radicalmente, ma mitigando gli effetti del degrado quando ai nostri occhi risultino indecorosi, ossia lesivi di quella che riteniamo essere la dignità formal e dell'edificio;

- tenere conto del luogo e del contesto.

\section{IL “CHIOSTRO DELLE CUCINE"NEL CONVENTO DI S. PAOLO A PARMA}

In certe situazioni è possi bile ricercare spazi per una musealizzazione protetta del'architettura, trattandola come un rudere interno $o$ un reperto inespl orato. II ridurre al massimo gli interventi sia conservativi che di restauro sulla materia stratificata può sembrare una rinuncia e un puro congelamento, ma in questi casi significa lasciare che la carica espressiva e documentaria di una testimonianza costruita, soprattutto quando già forte ed esplicita, possa continuare a sprigionarsi inal terata. Teniamo conto che si tratta di una scelta applicabile in un numero limitato di situazioni, in quanto richiede la convergenza di più fattori e la presenza di condizioni che la consentano.

$\mathrm{N}$ el caso della torre e della facciata della chiesa al tomedievale (X-XI sec.) inglobate nel convento di S. Paolo a Parma, questi oggetti sono entrati a far parte di un percorso museale (la Pinacoteca Stuard trasferita negli edifici attorno al chiostro) in cui improvvisamente si incontrano e si attraversano come relitti della storia, frammentari ma di grande significato e fascino.

G li interventi necessari al passaggio da parte dei visitatori sono separati fisicamente dalla materia antica, con la quale si ricerca un contatto "secco", non intrusivo e sem-

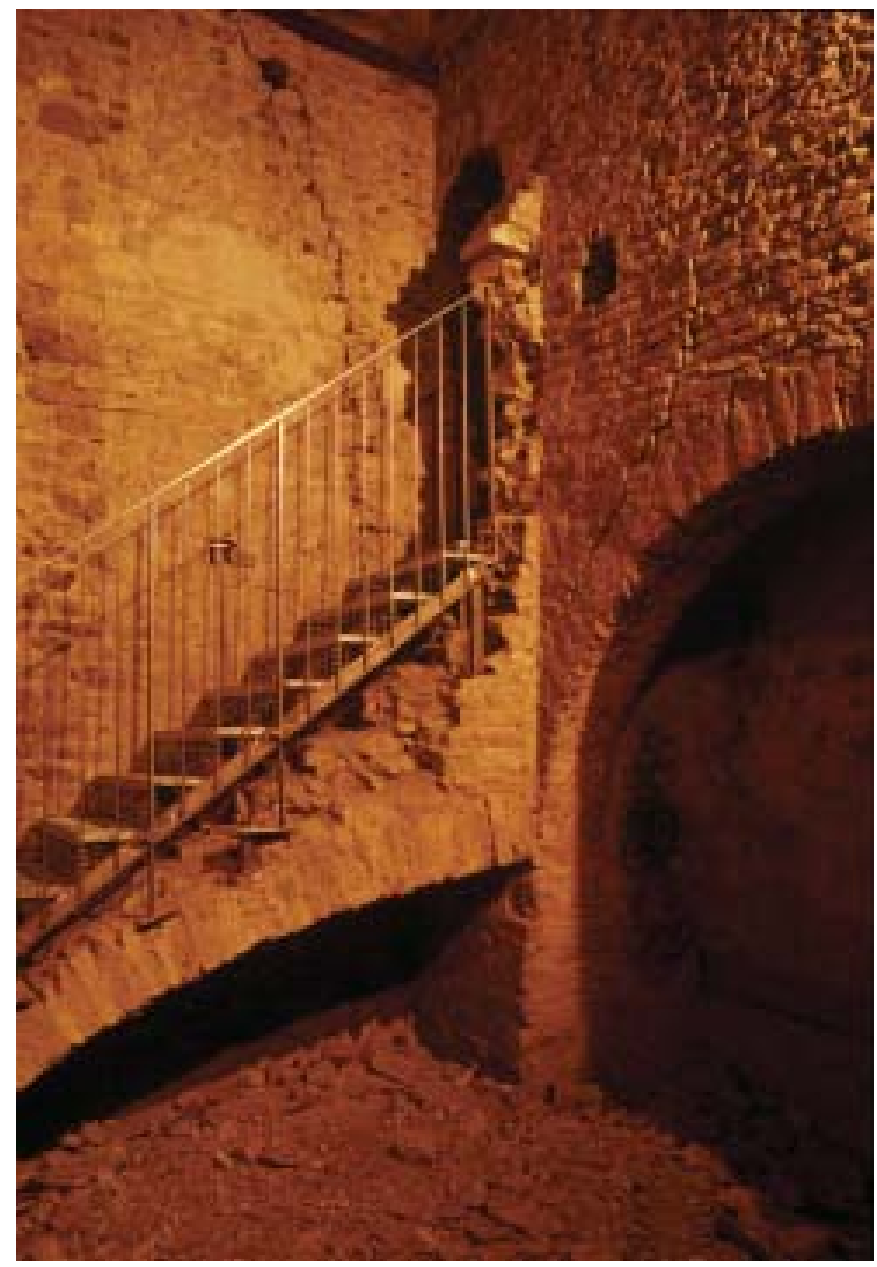

Fig. 2. La scala appoggiata attraversa la torre altomedievale mantenuta "a rudere interno". Convento di S. Paolo, Parma

pre rimuovibile: la scala in metallo che sale nell'ambiente della torre è appoggiata a quella antica (fig. 2), che ha perduto gli scalini, attraverso martinetti el evabili, fissaggi a ganascia, tiranti sospesi inseriti in buchi da ponte. Tutto questo consente di non ricostruire gli scalini invadendo il relitto dell'antica scala, di lasciare sul pavimento anche gli accumuli di detriti (coppi, mattoni, pietre), di non restaurare le superfici murarie, in cui sono solo stati fissati i conci pericolanti. La leggibilità stratigrafica e costruttiva è quella che il degrado, i crolli, le superfici "autentiche" consentono, senza ausili ricostruttivi, senza sottrazioni 0 selezioni. La scala può essere rimossa senza impatti sulla stratificazione iniziale, sulla quale rinuncia ad incidere. Un plastico ed alcuni pannelli grafici porgono al visitatore alcune spiegazioni e gli propongono interpretazioni sulla costruzione che ha di fronte.

In un'altra zona del complesso, la rimozione di una controparete ha riportato in luce una articolata superficie 


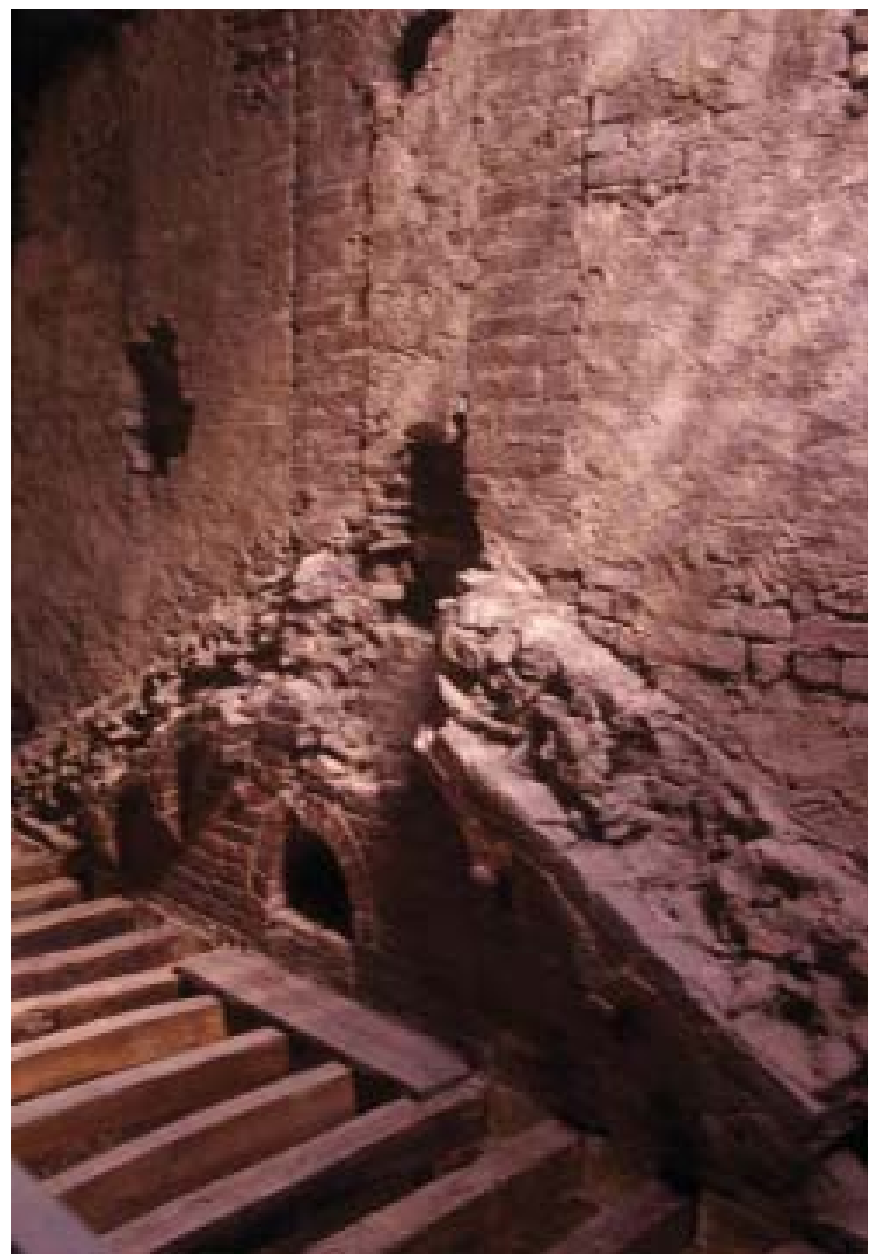

Fig. 3. La cuspide e la vela della facciata a nord del transetto inserite nel percorso museale con limitati interventi. Convento di S. Paolo, Parma

muraria, sulla quale si distinguono un affresco a fascia rinascimentale, i fori in cui erano inseritele travi del solaio coevo, i bordi di un camino stratigraficamente coerente con la superficie affrescata, e poi intonaci al grezzo, spalle di aperture murate ed al tre tracce. Anche in questo caso è stato pressochè omesso il restauro, se si esclude una blanda pulitura di tutta la superficie, senza distinzioni se dipinta o intonacata al grezzo, e al cune limitate stuccature di fissaggio. U na scala vi passa vicino, e la comprensione di trovarsi di fronte alla testimonianza di un diverso assetto è immediata e naturale (fig. 4).

Qui devo segnalare un aspetto. Molto spesso gli interventi di restauro di superfici che includono affreschi prestano la massima attenzione al testo pittorico, e considerano tutto ciò che non è dipinto (intonaci grezzi o rifiniti, murature, ecc.) come lacuna o assenza dell'affresco, trattandolo di conseguenza. Sono frequenti le ricoperture

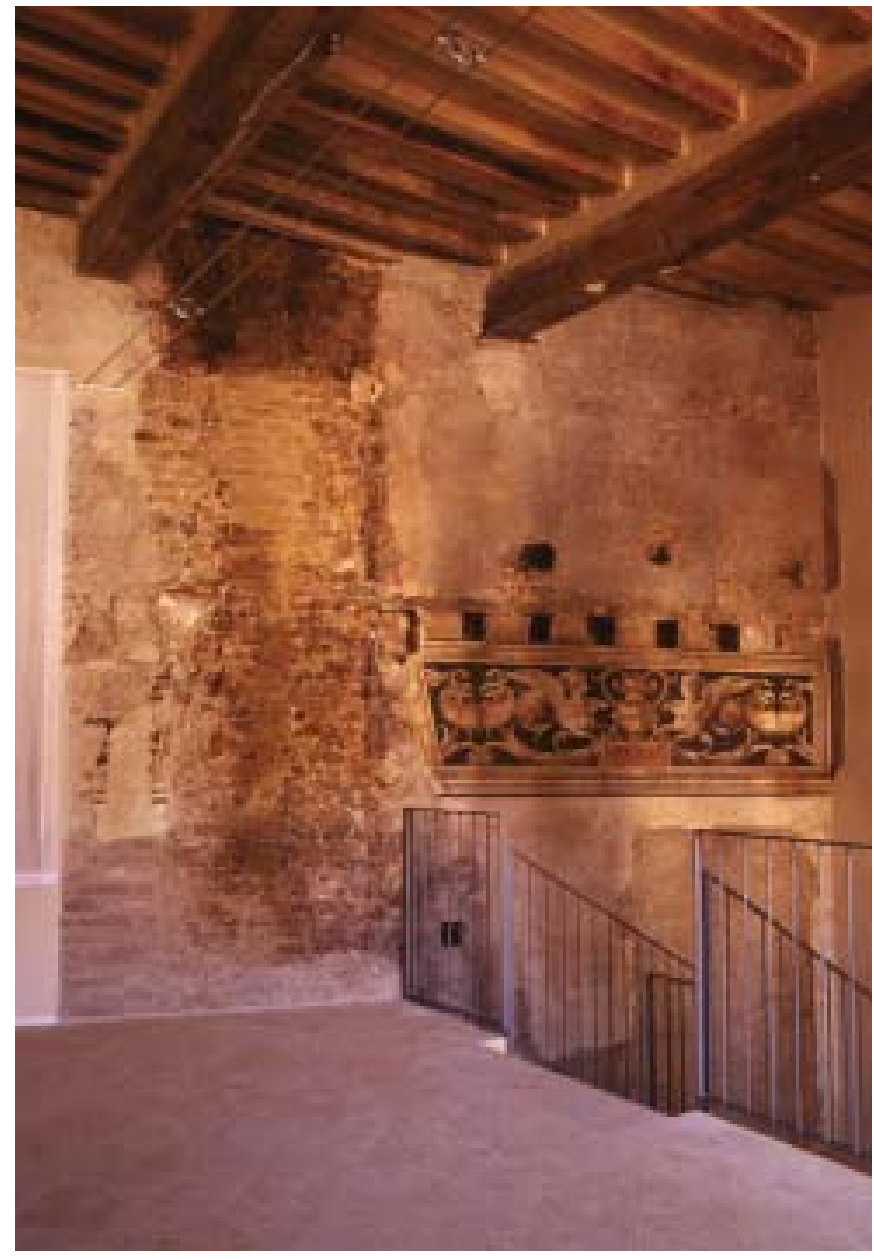

Fig. 4. La parete con le tracce del diverso assetto degli ambienti interni e la fascia rinascimentale, affiancata dalla scala di accesso che ne favorisce l'osservazione. Convento di S. Paolo, Parma

"neutre" a malta omogenea variamente trattata o le integrazioni pittoriche sottotono. Soprattutto quando lo strato di pinto è frammentario la conseguenza è grave, in quanto sotto il profilo stratigrafico-costruttivo questo intervento ha impatti di poco inferiori allo strappo dell'affresco e alla ricollocazione in al tro luogo. II contesto stratigrafico dell'affresco, quando non del tutto perduto a seguito della demolizione di parti al contorno ritenute "non interessan$\mathrm{ti}^{\prime}$, diviene comunque illeggibile e lo strato dipinto perde il nesso con la costruzione a vantaggio di un isolamento visibilista, preferito in genere dagli storici dell'arte, ma spesso anche da colleghi architetti.

N ell'ala restaurata del complesso di S. Paolo le parti mantenute "a rudere interno" sono state limitate alle zone sfuggite alle trasformazioni ottocentesche e maggiormente espressive e articolate (fig. 3). Coincidono sostanzialmente con quelle il cui carattere è riconducibile al primo 
gruppo di manufatti in base alla schematica suddivisione prima proposta, in un complesso che si presenta nell'insieme come fortemente riunificato, ossia il cui carattere rientra nel secondo gruppo, con altre parti che possiamo definire di transizione. II ricorso a testimoni isolati è stato ridotto, anche per condizionamenti esterni, a pochi elementi quali una monofora romanica e alcuni tratti di affresco o di muratura a vista con trattamenti antichi molto riconoscibili. Il loro significato è quello di dar prova di una lunga esistenza nel tempo della parte di costruzione in cui si trovano, che le trasformazioni avvenute ci fanno ora apparire in tutto diversa da come il singolo elemento suggerisce essere stata in passato. $\mathrm{N}$ on è tanto quindi una ostensione del reperto in sé, che inevitabilmente emerge come da una fossa di scavo nei paramenti recenti di cui turba la continuità, quanto il tentativo di mantenere caposaldi di riferimento della storia costruttiva: se la monofora romanica si trova a sette metri di altezza in un muro molto lungo che conserva a grande distanza altre testimonianze di quell'epoca, si può ritenere che l'intero muro fino a quella quota, ora non più osservabile perché ricoperto di intonaco, possa essere stato costruito in quella fase. $\mathrm{N}$ on è una prova compiuta, ma è assai più di un indizio, e questo ha conseguenze importanti sull'interpretazione della storia costruttiva dell'intero complesso. Gli studi stratigrafici compiuti da Paola Squassina nel corso del cantiere, dei cui risultati alcuni pannelli illustrativi danno conto restituendo una efficace interpretazione del processo formativo, costituiscono perciò il contesto di riferimento entro cui collocare e attribuire senso a questi el ementi sporadici (Doglioni, SQuassina, 2000). La stessa scelta degli elementi da mantenere a vista teneva conto in una strategia complessiva della capacità di ciascuno di testimoniare puntualmente un assetto o una fase costruttiva; i condizionamenti prima ricordati hanno reso maggiormente episodica la scelta.

In altri contesti, come nelle superfici dei muri di fondo e delle volte del chiostro, le superfici stratificate ed eterogenee sono state velate con una tinta di calce non del tutto coprente, che lascia in parte trasparire le caratteristiche materiche dei diversi strati. II risultato, nelle situazioni più felici, rappresenta per la superficie un punto di equilibrio tra restituzione di decoro attraverso una cromia relativamente omogenea, leggibilità stratigrafica, pur attenuata e resa più difficoltosa, mantenimento di una matericità sensibile che non tradisce il carattere del manufatto.

In un caso si è cercata la complessiva coerenza delle superfici all'interno di un ambiente, nel quale le superfici decorate a tempera nel XIX secolo sono prima state riportate a vista rimuovendo gli strati di scialbo sovrapposti, e successivamente restaurate.

Nello stesso complesso sono quindi stati utilizzati modi diversi e articolati di trattare le superfici stratificate, e il progetto ha tenuto conto in una certa misura dei risultati della ricerca stratigrafica, collocandoli tuttavia all'interno di una poetica fondata sull'aderire al carattere della fabbrica e sul permettere che la testimonianza di retta della sua materia giunga direttamente a chi la visita.

\section{LA CHIESA DI S. MARCELLO AD UMIN DI FELTRE}

Costruita nel XIII secolo affiancandosi al preesistente campanile di una chiesa demolita o crollata, ampliata poi nel XV, XVI e XVII secolo, la chiesa di S. Marcello non ha mai avuto una fase di unitaria riconfigurazione architettonica, continuando a presentarsi come somma di elementi eterogenei, condizione solo attenuata dalle intonacature ottocentesche.

$\mathrm{N}$ el corso degli anni '80 una campagna di saggi sugli intonaci recenti aveva segnalato la presenza sottostrato 0 direttamente riportato in luce vasti corredi pittorici, a partire dalla fase più antica fino al XVII secolo. Inoltre il paramento della muratura duecentesca, visibile dove erano caduti gli strati dipinti, presentava una accurata fugatura a giunto stilato, in parte ribadito ad affresco rosso. $\mathrm{N} \mathrm{el}$ corso degli anni '90 erano stati compiuti alcuni primi restauri nell'aula, insieme alla rimozione degli intonaci ottocenteschi. II progetto di restauro è intervenuto in una situazione "a scavo compiuto", ed ha operato una preliminare lettura stratigrafica delle superfici visibili. N ell'aula le diverse stesure di affresco si presentavano fortemente lacunose, ma soprattutto realizzate attuando programmi parziali, non unitari, che hanno portato ad accostare 0 sovrapporre nel tempo episodi pittorici diversi per soggetto e dimensione, facendo convivere gli strati di intonaco grezzo o parzial mente rifinito coevi o precedenti e creando una complessa e significativa articol azi one stratigrafica.

Sulla parete nord, ad esempio, I'U Itima Cena tardotrecentesca, il cui bordo superiore corrispondeva all'altezza della chiesa iniziale, viene inquadrata un secol o e mezzo dopo da un affresco rinascimental e raffigurante un loggiato aperto in prospettiva, dipinto sulla parte nel frattempo soprael evata della chiesa. La stesura cinquecentesca forma un "fal so bordo", o bordo accostato, rispetto al bordo superiore dell'U Itima Cena, fatto che testimonia, ol tre al rapporto stratigrafico di posteriorità, l'intenzione dell'affresco più recente di lasciare a vista quello più antico e di formare affiancandosi ad esso una nuova unità figurale ed una nuova interfaccia di periodo. 


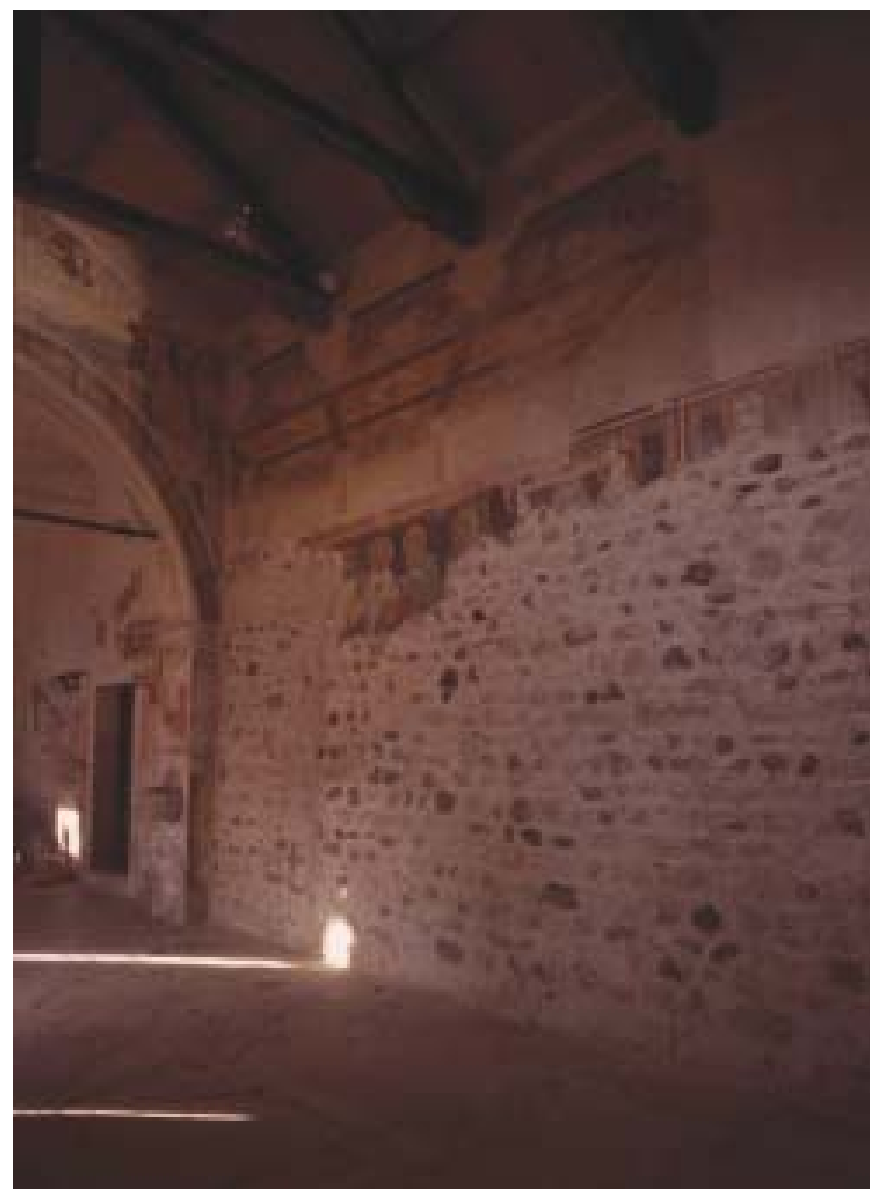

Fig. 5. Vista della parete nord dell'aula ad intervento compiuto. Nella parte di muratura a vista l'intervento è consistito nella pulitura dei giunti, con limitate risarciture. Chiesa di S. Marcello ad Umin, Feltre

Sulla parete sud, uno strato di intonaco grezzo prosegue in continuità stratigrafica con la stesura dipinta duecentesca, e gli affreschi di epoche successive vi si sovrappongono, a volte con i loro strati di intonaco non dipinto in continuità, che sovente saturano a filo le lacune delle stesure più antiche.

Rispetto al caso del S. Paolo a Parma, non è parsa applicabile una soluzione "a rudere interno", per più motivi: la superficie attuale è il risultato artificial e di uno "scavo" e dei primi interventi, e non presenta perciò la natural ezza propria del lungo degrado e dell'abbandono; inoltre la funzione richiede comunque un adeguato livello di decoro.

D'al tro canto, la frammentarietà degli strati di affresco rendeva comunque difficilmente praticabile una impostazione, se non ricostruttiva, comunque rivolta ad accentuare e reinquadrare la presenza degli affreschi presenti, attraverso integrazioni neutre o contorni sottotono.

A ttraverso il lavoro di un gruppo di restauratori, che hanno rivol to le proprie attenzioni tanto agli affreschi che

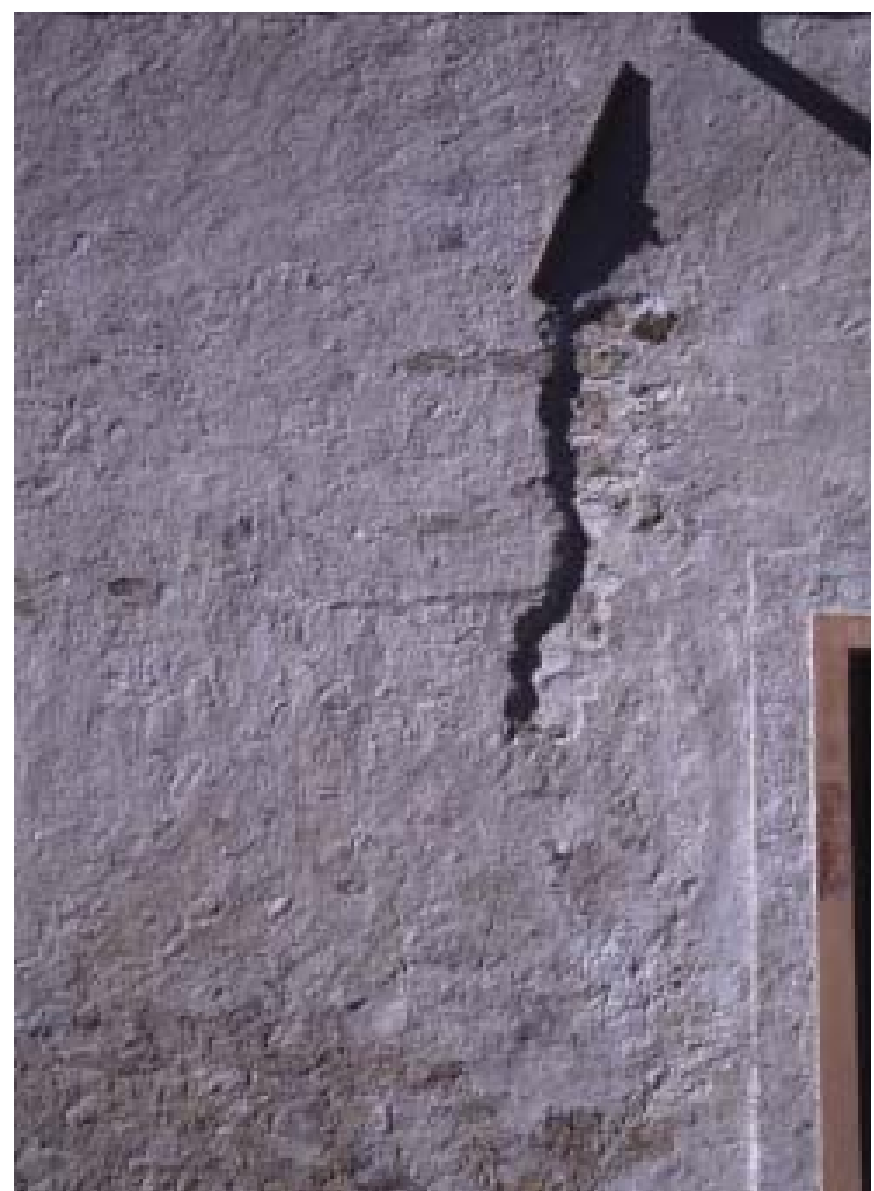

Fig. 6. Ad intervento concluso la luce radente mette in evidenza l'interfaccia negativa che corrisponde alla demolizione della facciata della chiesa romanica e all'ampliamento ed innalzamento della chiesa. Chiesa di S. Marcello ad Umin, Feltre

alle altre superfici (gli intonaci non dipinti, la muratura stilata), si è ricercata la compresenza dei diversi strati senza privilegiare le parti usualmente considerate più pregiate - come gli affreschi- rispetto alle altre, esponendole con natural ezza, riducendo al massimo i commenti e le separazioni. In questa impostazione, la muratura non è più il puro supporto di un'opera d'arte pittorica, el'affresco continua ad essere parte del contesto stratificato.

Descrivo brevemente alcuni degli accorgimenti adottati in questa opera, concordati e sperimentati con i restauratori e gli artigiani, ben consapevoli della delicatezza dell'opera:

- non sono state realizzate stuccature "salvabordo", con cui usualmente i restauratori consolidano al perimetro una lacuna di intonaco, e sono state sostituite da consolidamenti al contorno di minore impatto materico e visivo. La pratica del "salvabordo" ha l'effetto indesiderato di nascondere l'interfaccia negativa e di coprire il punto di contatto stratigrafico, rendendo al 
tempo stesso illeggibile la stratificazione e innaturale la superficie, in quanto introduce una azione estranea di rilevante impatto percettivo ed un nuovo vero bordo con proprio disegno del tutto casual e;

- per lo stesso motivo, sono state limitate al massimo le integrazioni di lacune, che pure si sono rese necessarie in alcuni punti all'interno o nell'immediato contorno degli affreschi;

- sono state il più possibile valorizzate le antiche stesure al grezzo, con le puliture o con il ricorso a velature di accompagnamento, ove utili ad ottenere superfici il cui minore scarto cromatico rispondesse al meno in parte alla richiesta di decoro;

- sono state limitate le integrazioni cromatiche delle superfici affrescate, per evitare, saturando il colore di queste, di accentuare l'effetto di frammentarietà e il distacco dalle superfici dipinte.

II restauro delle superfici dell'aula raggiunge a mio avviso un punto di equilibrio tra omogeneità cromatico-materica richiesta dalle istanze di decoro, e leggibilità natural e dei processi di costruzi one e modificazione (fig. 5).

Sulle superfici esterne della chiesa l'intervento ha rimesso in luce, consolidato e in parte integrato gli intonaci al grezzo presenti, ponendo particolare attenzione a mantenere leggibili le interfacce e i bordi stratigrafici dai quali si comprendeva con chiarezza il processo di accrescimento in al meno tre fasi della chiesa (fig. 6).

\section{LE SUPERFICI DELL'EX-CONVENTO DEI SS. COSMA E DAMIANO A VENEZIA}

Costruito tra la fine del XV e i secoli XVI-XVII, il convento dei Santi Cosma e Damiano era abbandonato da quasi trent'anni e gravemente danneggiato dal crollo delle coperture. Del restauro compiuto esaminiamo distintamente due temi, il primo relativo alle superfici dei fronti esterni, il secondo alle superfici dei grandi ambienti delI'ala monumentale.

R ealizzato in età in cui, a Venezia, il rivestimento ad intonaco delle murature esterne in mattoni è pratica prevalente, il complesso aveva conservato sui fronti solo a tratti la finitura antica, in quanto il dilavamento el'umidità di risalita unita alla salinità avevano causato, soprattutto nell'ultimo periodo, degrado e larghe perdite degli intonaci, parte a marmorino e parte a cocciopesto.

Sulla muratura messa a nudo si leggevano le tracce, non particolarmente articolate, dei processi di trasformazione. La scelta di restauro, tuttavia, è stata solo in subordine rivolta a mantenere la leggibilità di questi segni, e si è indirizzata alla conservazione dé tratti superstiti di intonaco e alla complessiva ricostruzione del paramento perduto.

Espongo le ragioni di questa scelta.

Venezia sta subendo, anche sulla spinta dei fenomeni indotti dal turismo internazionale, un rapido e poco controllato mutamento delle superfici, dovuto in particolare alla diffusa sostituzione di paramenti di intonaco spesso antichi con al tri nuovi, che impiegano materiali e tecniche moderne. Per di più, il tentativo di mantenere una somiglianza con gli intonaci sostituiti porta frequentemente ad esiti inadeguati quando non caricaturali e produce un rapido mutamento di immagine della città. Se da un lato diventa importante, in questa fase di drastica rarefazione delle testimonianze autentiche, opporsi alla sostituzione degli intonaci antichi conservandoli il più possibile, dalI'altro è necessario comunque trovare i mezzi per esaudire la richiesta di decoro e di rinnovo che proviene dalla collettività e dalla committenza, che spinge a rinnovare le superfici per cancellarne il degrado ed esorcizzare così l'idea stessa del decadimento della città. E' necessario perciò saper coniugare la conservazione del le testimonianze con la restituzione del decoro al la costruzione.

L'impostazione architettonica dei fronti del Convento di S. Cosma e Damiano è basata sulle cornici in pietra d'Istria 0 in marmorino bianco sui fondi in cocciopesto rosato. II paramento costituisce quindi una parte fondamentale del linguaggio, del cromatismo e della configurazione architettonica dell'edificio, e la sua disarticolazi one o perdita totale conseguente all'accettazione dell'attuale frammentarietà dei rivestimenti rappresenterebbe un totale cambiamento. Rispetto ai due gruppi prima schematizzati, I'edificio si trovava in una condizione mediana: nel secondo gruppo per la configurazione architettonica unitaria e regolare, nel primo per le eterogeneità materiche prodotte dal degrado. La scelta è stata di privil egiare l'appartenenza architettonica al secondo gruppo, mantenendo la quota di eterogeneità conseguente alla conservazione dei tratti antichi di intonaco e alla contestuale ricostruzione degli intonaci perduti. Si è voluto affermare, con questo, la possibilità anche a Venezia di contrastare gli effetti visivi del degrado restituendo una immagine decorosa e nel suo insieme unitaria, senza per questo cancellare del tutto i segni del passaggio del tempo, e conservando accuratamente i tratti di paramento antico, puliti e consolidati.

Sotto il profilo stratigrafico, il risarcimento dell'intonaco è avvenuto a fil o formando un fal so bordo con le parti antiche conservate, la cui interfaccia è stata mantenuta perfettamente visibile attraverso una accurata pulitura della zona di contatto (fig. 8). In alcuni tratti, I'intenzio- 


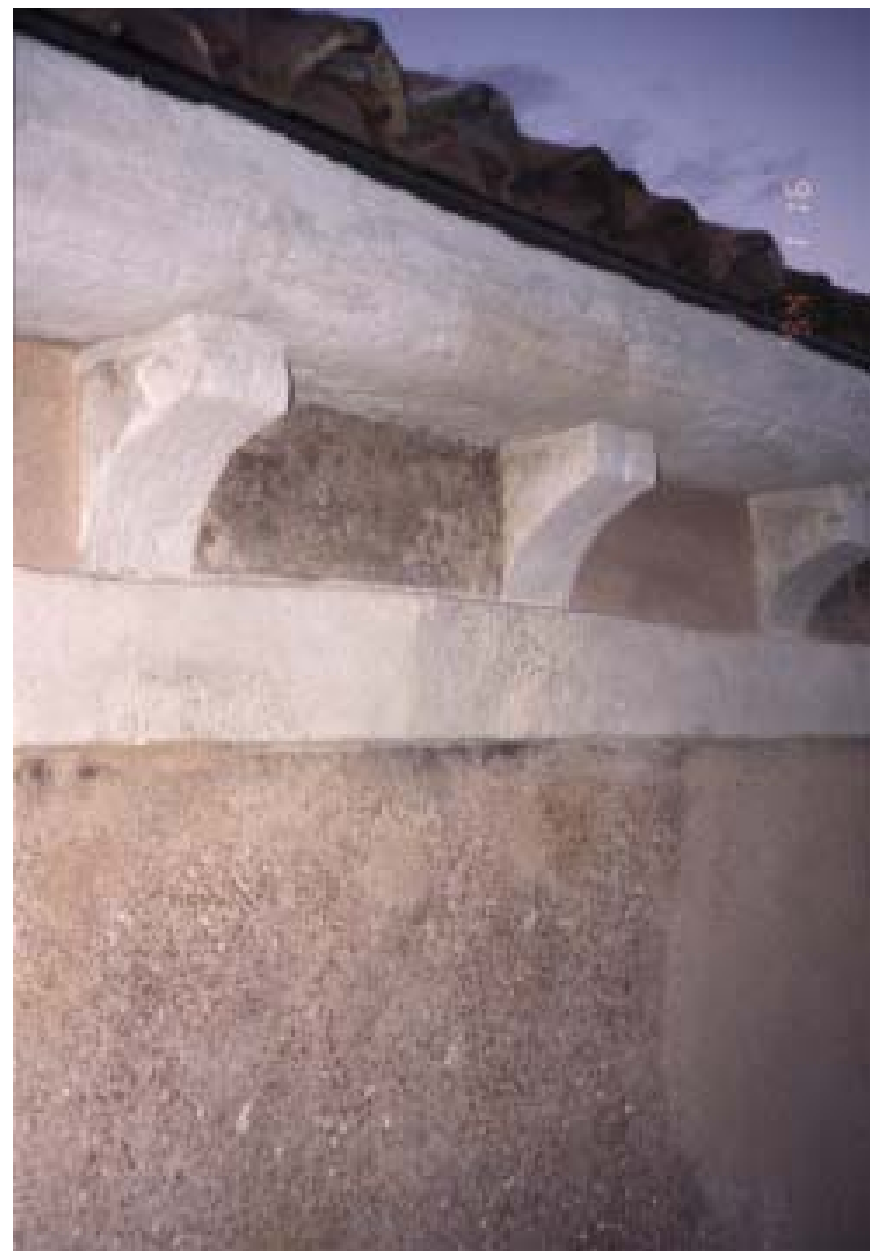

Fig. 8. Particolare dell'intonaco antico dopo la pulitura, con le integrazioni a filo. Fronte esterno del convento di S. Cosma e Damiano, Venezia

nale riduzione di spessore del nuovo intonaco lascia trasparire la tessitura muraria sottostante ove questa presenta elementi particolarmente significativi, come la traccia di un grande camino. Questo ha consentito di raggiungere una complessiva omogeneità cromatica, funzi onal e a restituire unitarietà architettonica ai fronti, e al tempo stesso ha permesso di lasciare intuire, attraverso l'articolazione della tessitura, la presenza e la natura degli el ementi architettonici non più in uso (fig. 9).

Si è posto diffusamente un problema, di ordine sia pratico che concettuale. La scel ta di conservare i tratti ancora esistenti di intonaco e di integrarne le mancanze con uno strato cromaticamente e matericamente affine, trovava ostacolo sia nel degrado differenziato che i tratti antichi presentavano nelle diverse parti, sia nella omogeneità e rigidità che inevitabilmente un nuovo intonaco presenta rispetto all'antico variegato dal degrado. L'adozione di un

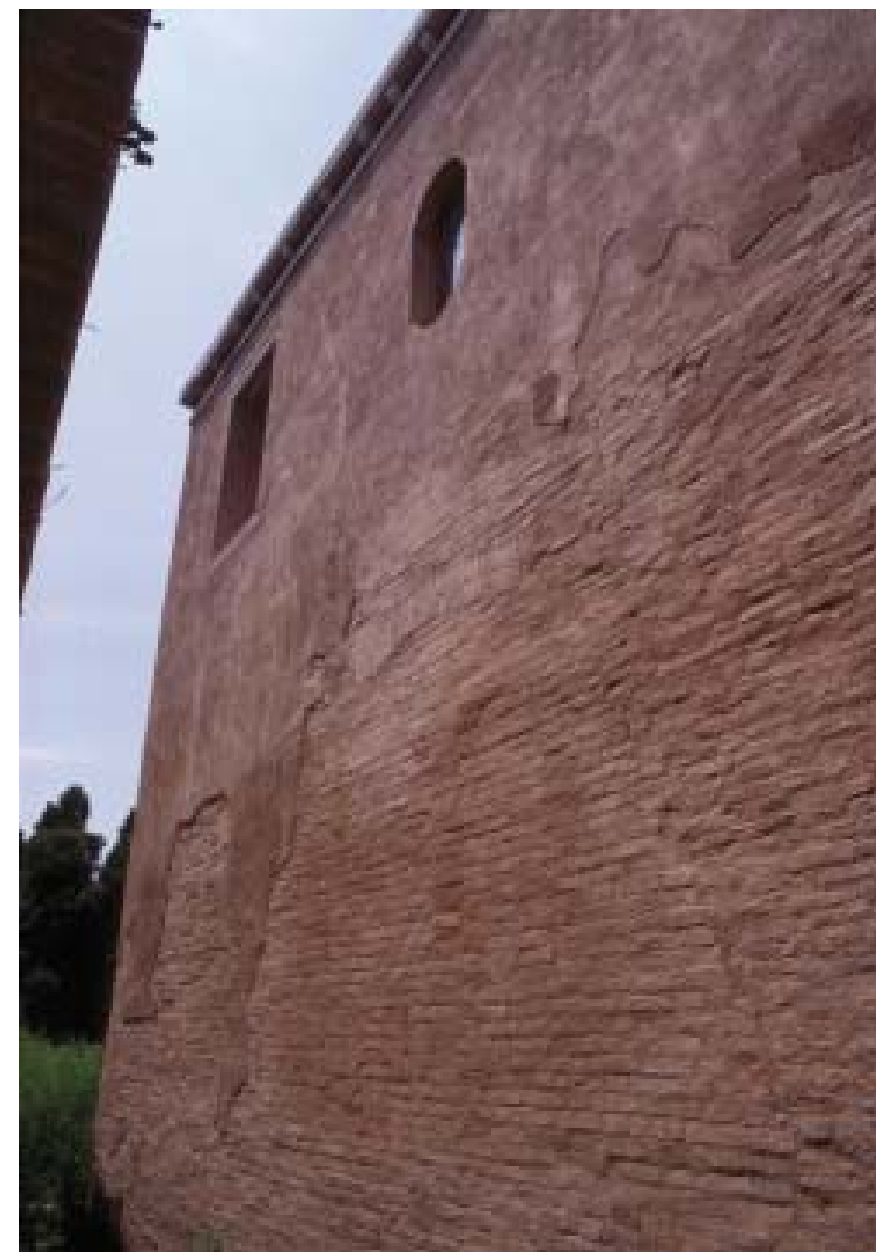

Fig. 9. Trattamento di intonaco a strato sottile, steso a pennello, a raccordo cromatico con l'intonaco antico, per mantenere osservabile la tessitura muraria e le tracce presenti. Fronte laterale del convento di S. Cosma e Damiano, Venezia

unico tipo di intonaco di integrazione e di un unico trattamento di superficie avrebbe prodotto un risultato a "pelle di leopardo" per le differenze anche forti tra zona e zona e per l'emergere del nuovo intonaco rispetto all'esistente (fig. 7). Si è adottato quindi un criterio "di prossimità", accentuando la pulitura ed eventuali limitati risarcimenti di superficie dei tratti di intonaco conservato che si erano allontanati troppo, matericamente o cromaticamente, dalla condizione media; simmetricamente negli intonaci nuovi è stata real izzata una particol are lavorazione del la superficie, attraverso lavaggi accentuati o ridotti che, riportando in evidenza maggiore o minore l'inerte in cocciopesto o in pietra d'Istria dell'impasto, permettevano di ridurre le differenze più marcate rispetto ai contigui intonaci antichi in cui il dilavamento aveva prodotto nel tempo un effetto simile. L'integrazione è stata cal ibrata in rapporto allo stato attual e preval ente delle parti superstiti e non su uno stato 


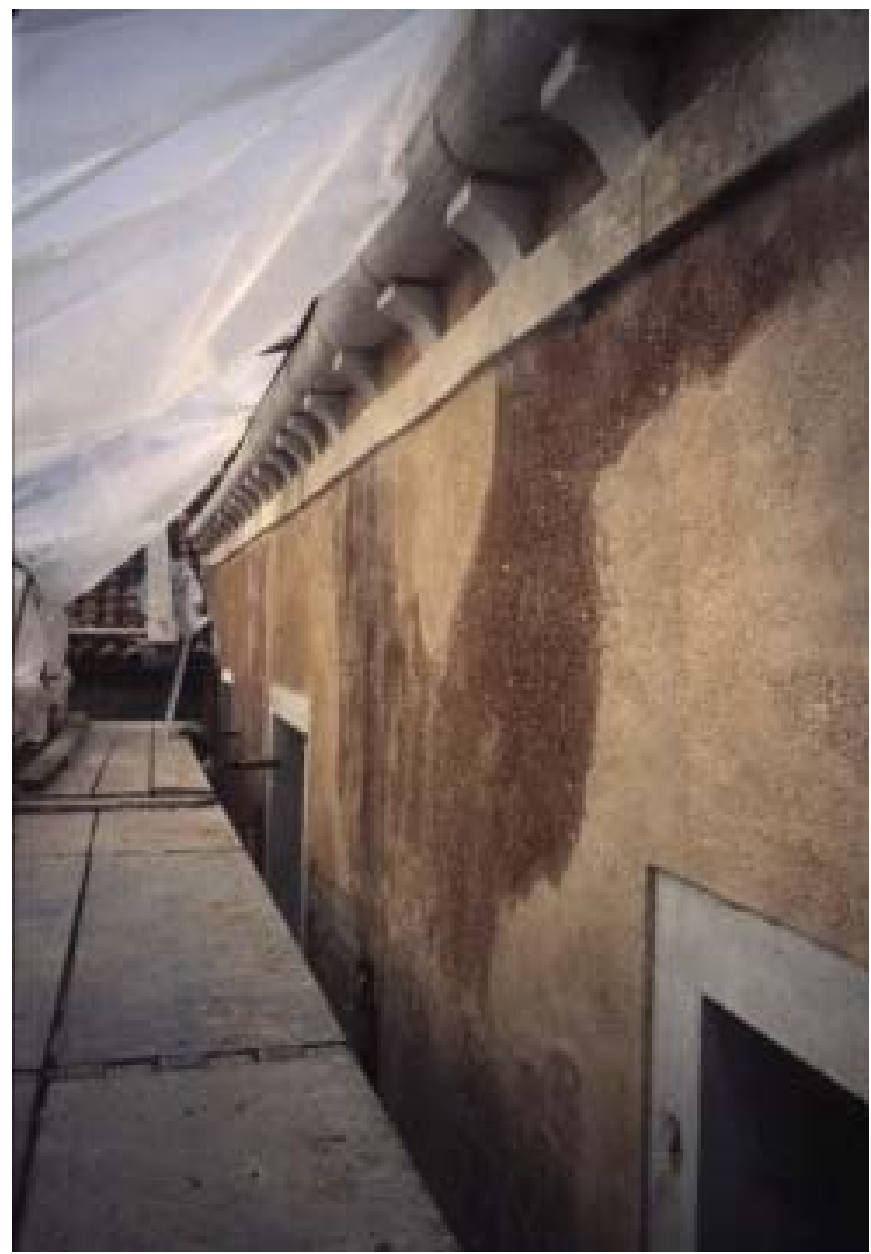

Fig. 7. L'effetto "a pelle di leopardo" dei tratti di intonaco antico, dopo l'integrazione delle parti perdute e prima dei trattamenti. Fronte esterno del convento di S. Cosma e Damiano, Venezia

supposto iniziale, chei campioni conservati in aree più protette ci indicavano come notevolmente diverso.

La blanda imitazione degli effetti di degrado - in certa misura questo è stato fatto- ha favorito la conservazione delle parti antiche, le ha comunque mantenute distinguibili eal tempo stesso ha restituito unitarietà cromatica e materica al fronte nel rispetto della tradizione veneziana, soddisfacendo le richieste di decoro. Parigi val bene una M essa?

L'intervento sulle superfici interne dei grandi ambienti ha assunto finalità e modi esecutivi in parte diversi ${ }^{2}$. Alla ricerca di unitarietà sia pure articolata nei fronti esterni, si è qui preferito lasciare maggiore spazio al racconto della materia sulle pareti, sulle quali rimangono

${ }^{2} L$ 'intervento sulle superfici interne del Convento di $\mathrm{S}$. Cosma e Damiano è già stato descritto in BRUSCHETTI, DOGLIONI, 2000: 231-238, che qui si riprende in parte e cui si rinvia per maggiore completezza. leggibili elementi che sono stati invece ricoperti sui fronti e che permettono quindi una lettura complementare.

II crollo di buona parte delle coperture aveva determinato un gravissimo degrado sulle pareti, ancora parzialmente ricoperte da un intonaco real izzato nella fase in cui il complesso era stato trasformato in magazzino militare. Si è decisa e realizzata la rimozione di questo strato con modalità tali da non danneggiare le superfici sottostanti, sulle quali sono emerse le tracce degli assetti precedenti. Sono comparsi tra l'altro i segni di appoggio di un soffitto a volta ora perduto e, ancora, i segni delle fasi di modificazione delle aperture ad arco verso nord e delle porte verso la chiesa, insieme alla presenza di lunettoni murati nella parte al ta della parete verso sud.

L'interpretazione delle fasi costruttive a seguito delI'analisi stratigrafica ha portato ad identificare al meno due assetti di grande coerenza: un primo, forse tardoquattrocentesco, con copertura a quota inferiore rispetto all'attuale, segnalata da una lunga rema lignea che funge anche da architrave ad una serie di finestre quadrate sui fronti sud e nord; un secondo, sei-settecentesco, in cui l'aula è stata innalzata aprendo i lunettoni verso sud i quali, insieme alle finestre ad arco a nord, che ampliavano le precedenti, si inserivano nelle unghiature di una volta a schifo lunettata, delle cui centine lignee, sostenute nella parte centrale dalle capriate del tetto, sono rimasti chiari segni di appoggio sulle murature. Doveva trattarsi di un assetto molto forte, in cui la sala, inizial mente lunga $40 \mathrm{~m}$. e larga 11 , ora suddivisa in due ambienti dalla presenza di un muro mediano, risultava unificata soprattutto dal soffitto a volta. $\mathrm{Ma}$, a parte alcune aperture, ancora utilizzate $\mathrm{O}$ murate, di questa configurazione restavano solo interfacce negative, segni di assenza, come le tracce di imposta delle volte, più che presenze fisiche (fig. 10-11).

Il progetto di restauro è stato reindirizzato sulla base delle superfici emerse e della loro lettura stratigrafica. Possiamo cosi riassumere sinteticamente gli obiettivi ed i criteri adottati. Sotto il profilo strettamente stratigrafico si è cercato di:

- mantenere la leggibilità stratigrafica diretta dei bordi (veri bordi, falsi bordi, interfacce... );

- non operare selezioni tra le tracce, e mantenere la compresenza dei diversi assetti;

- evitare per quanto possibile la formazione di nuovi veri bordi. è inteso:

Sotto il profilo della configurazione architettonica si

- formare un nuovo contesto in cui siano evidenti alcuni elementi riunificanti: vedi ad esempio la maggiore 


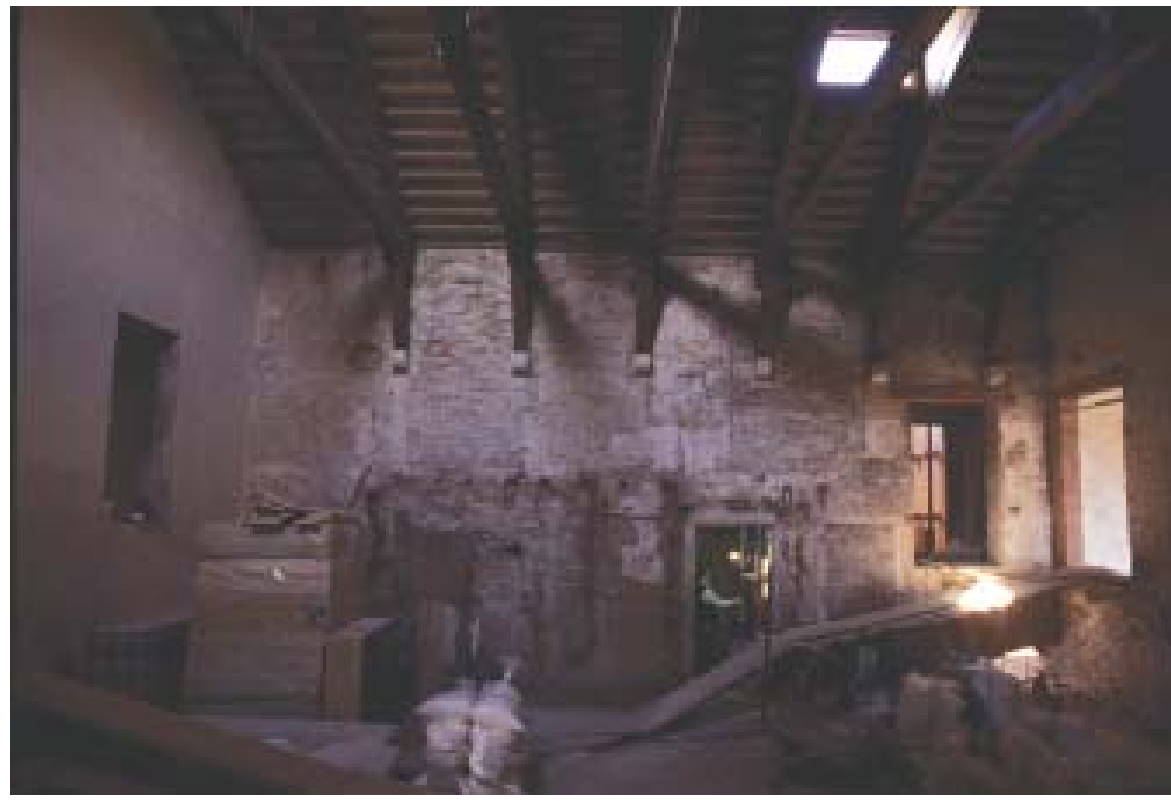

Fig. 10-11. Parete nord dell'ambiente centrale nel corso degli interventi (foto 10) e ad interventi ultimati (foto 11). Si osservano in particolare le grandi finestre murate dell'assetto seicentesco. Convento di S. Cosma e Damiano, Venezia

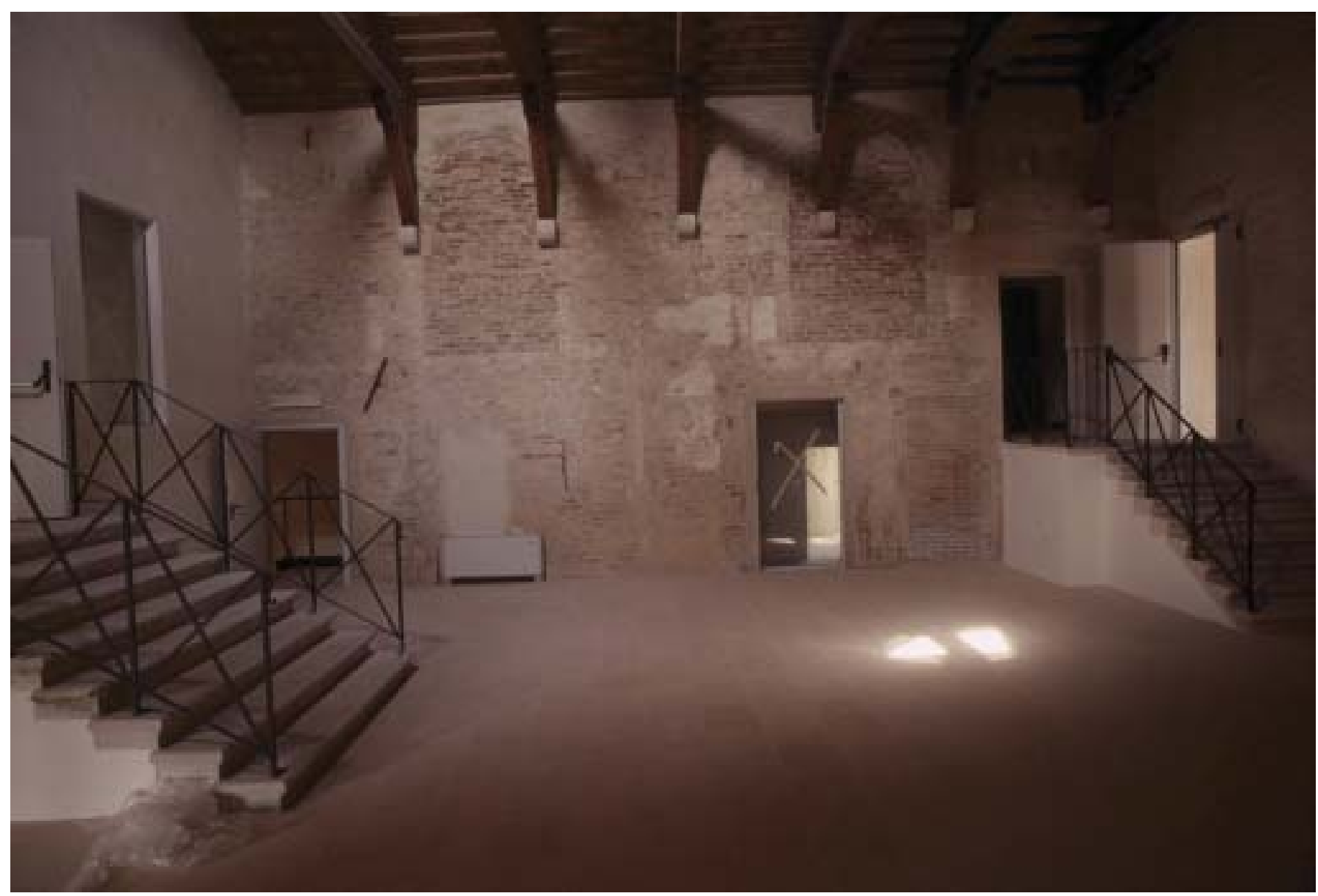


ricopertura ad intonaco chiaro delle parti inferiori, il mantenimento a vista degli elementi lineari costituiti dalle reme in legno, una relativa accentuazione per differenza della traccia di appoggio della volta perduta; a questo obiettivo di riunificazione contribuiscono anche il nuovo pavimento, in cocciopesto rosso, e il tetto a vista; - consentire una percezione spaziale di insieme della stratificazione, utilizzandone le potenzial ità espressive per articolare le vaste superfici prive di aperture e consentendo, con la modulazione dei trattamenti, di associare più facilmente ciascun elemento ad altri affini presenti su pareti opposte $o$ in posizione molto lontana.

Infine, sotto il profilo della funzionalità d'uso si è cercato di:

- rendere più compatta e stabile la superficie, ad evitare la caduta di frammenti e la formazione di polvere;

- rendere complessivamente più chiare le superfici, tenuto conto della ridotta illuminazione naturale dell 'ambiente.

Come si vede, gli obiettivi di progetto sono tra loro eterogenei, e presentano componenti di inevitabile contrasto. L'impegno si è quindi spostato alla ricerca e sperimentazione di modi operativi in grado di contemperare diverse esigenze, senza contraddire alcuni obi ettivi a favore di altri.

Le superfici murarie emerse dopo la rimozione dell'intonaco recente apparivano per la maggior parte essere state ricopertein antico da strati di finitura poi perduti. Lemurature della fase più antica presentavano una superficie più accurata e regolare, mentre erano in genere più irregolari e prive di piani quelle successive, in particolare i tamponamenti. I giunti di malta erano sgranati per buona parte della superficie, a seguito di rimozioni avvenute in antico, o comunque sommari e non rifiniti. Data questa situazione, si è ricercata una modalità di trattamento delle superfici articolata secondo il loro diverso carattere, in base a criteri fissati in via generale. $\mathrm{Ci}$ era ben chiaro che ogni intervento su questo palinsesto stratificato poteva costituirne una manipolazione se non uno stravolgimento. Indirizzare cia scuna operazione in modo da salvaguardare il dato stratigrafico presente nelle tracce ha costituito perciò il punto di partenza nella sperimentazi one del modo di operare.

Si è ricercata in primo luogo una modalità operativa che, pur consentendo la lettura dei nessi stratigrafici al perimetro delle unità, non li enfatizzasse forzandoli in modo innaturale. Perciò l'intervento sui bordi tra superfici diverse, pur raccordato a quello sulle superfici, mantiene sempre una sua specifica articol azione. Ad esempio, lo strato di ricopertura di una superficie si assottiglia progressivamente in corrispondenza del perimetro, sia esso costituito da interfaccia negativa o bordo, fino a diventare una velatura senza strato. Si tratta di una stesura a tinta leggera che raccorda cromaticamente il perimetro, dove si concentra una parte rilevante dei dati stratigrafici, alla superficie, senza ricoprirlo e lasciandone trasparire la materialità.

Si è sviluppato inoltre il ragionamento sul significato di alcune superfici murarie nella evoluzione della fabbrica. $\mathrm{N}$ on per trarne meccaniche corrispondenze - ciò che era a vista tale deve rimanere, ciò che era intonacato deve essere ricoperto- ma per aprire una possibilità all lusiva utilizzabile sia per l'aspetto della comprensione stratigrafica che per la formazione di un nuovo assetto architettonico. Ad esempio, la muratura al di sopra della fascia di imposta della vol ta in cannucciato non aveva presumibilmente mai avuto una superficie finita, e si prestava a rimanere a vista, quasi indisturbata, se non fosse per la presenza delle parti di muratura ricostruita nel corso degli interventi sul tetto. N ella zona al ta, dove si trovano anche i lunettoni e le tracce di appoggio delle centine lignee, si è scelto di ricoprire le sole parti di muratura nuova in corrispondenza dell'appoggio del tetto, limitandosi a risarcire i giunti nella parte sottostante fino al la fascia. II cambiamento di trattamento, I'arrestarsi della ricopertura parziale così realizzata corrisponde alla discontinuità costruttiva, e si raccorda a sfumare senza creare un nuovo bordo. Q uella che viene identificata come fascia di imposta del soffitto a volta, ossia l'interfaccia negativa rimasta dopo la caduta dell'intonaco a cocciopesto e marmorino di cui era costituita, viene conservata senza interventi, esclusa una blanda pulitura e fissatura. La sua impronta, formata da tracce di cocciopesto e da alcuni bordi presenti nell'intonaco conservato, costituisce un elemento architettonico assente, che lega insieme quasi l'intero ambiente. La scelta di operare su di esso in stretti termini conservativi si accompagna ad una sua accentuazione per differenza rispetto alle superfici contigue, che invece vengono velate o parzialmente coperte. La mancanza di trattamento vuole alludere, appunto, alla mancanza fisica della fascia, e al tempo stesso la pone in evidenza consentendo ad essa di svolgere un ruolo architettonico di articolazione della parete, suddividendola in una parte inferiore, storicamente finita ad intonaco, e in una parte superiore, con paramento murario al grezzo. II ruol o architettonico si sviluppa anche nell'intero ambiente, nel momento in cui consente di associare i lunettoni murati a sud con le finestre ad arco ancora aperte a nord, oppure esclude dall'assetto corrispondente alla presenza della volta la parete mediana verso est, più recente e perciò 
priva della fascia, parete che ora divide in due l'ambiente un tempo unitario.

N ella parte sottostante alla fascia, che delimita le superfici inizial mente intonacate di un unico ambiente, di al tezza inferiore nell'assetto iniziale, poi innalzato nell'assetto vol tato, si è cercato di raggiungere un maggior livello di finitura, ricoprendo la superficiea mattoni fino a raggiungere il raccordo a filo con i tratti di intonaco superstite. Tuttavia questo strato di malta chiara, sottile ed omogeneo, è steso in modo da conformarsi al supporto, quasi a diventarne parte facendone trasparire I'apparecchiatura. Si è cercato, attraverso stesure di malta fluida data parte a cazzuola, parte a pennello e successivamente lisciata, un modo per coprire solo parzial mente la muratura, lasciandola leggibile in trasparenza. A nalogamente, è stata operata la risarcitura solo in profondità delle discontinuità, di cui restano visibili i bordi: veri bordi, formati da spalle, ma anche interfaccia negative, dovute a rottura. II risultato è una risarcitura sottosquadro delle discontinuità nelle parti più interne, mentre il bordo in superficie rimane material mente visi bile e interpretabile sotto il profilo stratigrafico.

L'intervento ha preso forma nella sperimental ità del lavoro - campionature di lavorazioni su superfici e su nodi stratigrafici- ed ha avuto un momento di sintesi nella mappatura degli interventi, sovrapposta e connessa alle perimetrazioni indicate dalla lettura stratigrafica, in modo da fissare gli obbiettivi progettuali parte per parte e consentire agli operatori di riconoscere e applicare i diversi gradi e modi di intervento rispetto ad una data intenzione progettuale. Si è cosi definito un lessico operativo legato alla gra dual ità del le ricoperture che si vol evano raggiungere, e al la segnal azione delle particol ari modal ità di trattamento delle tracce più fragili e labili, che rischiavano di essere perdute se l'operatore, avvertito, non avesse prestato una specifica attenzione. Le maestranze, dopo un primo avvio con inevitabili difficol tà, sono progressivamente entrate nello spirito del lavoro, proponendo esse stesse soluzioni e adatta menti per raggiungere gli obiettivi indicati.

\section{Bibliografia}

BELLINI A., 1983, Istanze storiche e selezione nel restauro architettonico, Restauro, n. 68-69.

BONELLI R., 1986, Archeologia stratigrafica e storia dell'architettura, A rchitettura, storia e documenti, 2, pp. 5-10.

BRogiolo G. P., 1988, A rcheologia dell'edilizia storica, Como.

BRUSCHETTI A., DOGLIONI F., 2000, Le superfici dei grandi ambienti nel convento di SS. Cosma e Damiano a Venezia, A rcheol ogia del 'architettura, IV, pp. 231-238.

CristinelLi G., 2000, Relazione di apertura, in G. Cristinelli, V. FORAMITTI (a cura di), II restauro tra identità eautenticità, A tti della tavola roton- da "I principi fondativi del restauro architettonico", Venezia, 31 gennaio-1 febbraio 1999, Venezia.

D OGLIONI F., 1988, La ricerca sulle strutture edilizie tra archeologia stratigrafica e restauro architettonico, in Fran COVICH R., PARENTI R. (a cura di), A rcheologia e Restauro dé monumenti. I ciclo di lezioni sulla ricerca applicata in archeologia. Certosa di Pontignano (Siena) 28 settembre 10 ottobre 1987, Firenze, pp. 223-247.

DOGLION I F., 1993, Importanza dell'intonaco per la conservazione delle autenticità edilizienel restauro architettonico, in International W orkshop on conservation of architectural surfaces: stones and wall covering, Venice, 12/14 march 1992, Atti, Venezia.

D OGLION I F., 1997, Stratigrafia eR estauro- Tra conoscenza e conservazione del 'architetura, Trieste, pp. 1-312.

DogliON I F., SQUASSIN A P., 2000, Superfici stratificate in un contesto museale. II restauro del Convento di S. Paolo a Parma, A rcheologia del'architettura, IV, pp. 223-230.

PARENTI R., 1985, La torre A: una lettura stratigrafica, A rcheol ogia M edievale, XII, pp. 417-437.

QUENDOLO A., 2000, Riflessioni sul concetto di autenticità nel'attuale dibattito italiano sul restauro architettonico, tesi di Dottorato di Ricerca in Conservazione dei Beni Architettonici, XI ciclo, Politecnico di Milano.

TORSELLO P., 1988, La materia del restauro, Venezia, pp. 1-207.

TRECCAN I G. P., 2000, Archeologie e restauri, in TRECCANI G. P. (a cura di), A rcheol ogie, restauro, conservazione. M entalità e pratiche dell'archeologia nel'intervento sul costruito, Milano, pp. 11-97. 